\title{
REVIEW
}

\section{Sleep Optimization and Diabetes Control: A Review of the Literature}

\author{
Teresa Arora $\cdot$ Shahrad Taheri
}

To view enhanced content go to www.diabetestherapy-open.com

Received: September 1, 2015 / Published online: November 4, 2015

(C) The Author(s) 2015. This article is published with open access at Springerlink.com

\section{ABSTRACT}

Pre-diabetes and diabetes occur secondary to a constellation of pathophysiological abnormalities that culminate in insulin resistance, which results in reduced cellular glucose uptake and increased glucose production. Although pre-diabetes and diabetes have a strong genetic basis, they are largely environmentally driven through lifestyle factors. Traditional lifestyle factors such as diet and physical activity do not fully explain the dramatic rise in the incidence and prevalence of diabetes mellitus. Sleep has emerged as an additional lifestyle behavior, important for metabolic health and energy homeostasis. In this article, we review the current evidence surrounding the sleep-diabetes association.

T. Arora $\cdot$ S. Taheri $(\bowtie)$

Department of Medicine, Weill Cornell Medical College in Qatar, Room C008, Qatar Foundation, Education City, PO Box 24144, Doha, Qatar

e-mail: szt2004@qatar-med.cornell.edu

T. Arora

Department of Medicine, Weill Cornell Medical College, New York, USA
Keywords: Circadian

misalignment; Chronotype; Obesity; Sleep quality; Sleep duration; Type 2 diabetes mellitus

\section{INTRODUCTION}

The onset, progression and management of type 2 diabetes mellitus remain a major challenge. Given the many accompanying complications and comorbidities of diabetes, a comprehensive understanding of all factors underpinning its development and progression is absolutely essential. A profusion of literature surrounds the role of sleep and type 2 diabetes. Sleep is a significant and modifiable lifestyle behavior. Understanding the relationship between sleep and diabetes mellitus is crucial and will enable the development of strategies to improve the life of those with diabetes.

Sleep is regulated by two interconnected processes, named Process $\mathrm{S}$ (homeostatic drive) and Process $\mathrm{C}$ (circadian drive) [1]. Process $\mathrm{S}$ is appetitive in that a sleep debt occurs throughout the day and this increases the pressure to sleep. This sleep debt is repaid once sleep occurs. If the sleep debt is not sufficiently repaid, it accumulates, resulting in 
poor daytime function and potentially metabolic abnormalities. Process $S$ is associated with central adenosine accumulation. Caffeine is an adenosine receptor blocker. Therefore, caffeine intake around bedtime will reduce the homeostatic drive and reduce sleep duration. If Process $S$ were the only regulator, then because of accumulated sleep debt, one would fall asleep in the early evening. This is prevented by Process $\mathrm{C}$, which determines the timing of sleep. Circadian regulation occurs via the hypothalamic suprachiasmatic nucleus and is synchronized by light via the retino-hypothalamic tract. Thus, light exposure near bedtime delays sleep, and if this is combined with early morning awakening for work, then sleep duration is reduced. Many hormones are released in a circadian manner, a typical example being cortisol, which is high in the morning and low in the evening. If Process $S$ and Process $\mathrm{C}$ are desynchronized, as occurs in jet lag or night shift work, then metabolic consequences can ensue, secondary to hormonal alterations. Once sleep occurs, several hormones are released specifically linked to sleep stages. For example, growth hormone and prolactin are released during the deep stages of sleep. Thus, sleep loss can also impact the release of hormones that regulate metabolic function. Sleep is assessed using several approaches, each with its own advantages and disadvantages (Table 1).

The aim of this review is to highlight and discuss the relevant literature surrounding the relationship between sleep parameters and diabetes outcomes. Specifically, we provide a comprehensive review of the literature for multiple components of sleep (quantity, quality and timing, circadian misalignment and daytime sleep) and diabetes outcomes, which we present according to study design (case-control, cross-sectional, prospective and experimental). We further discuss potential mechanisms, limitations of the current literature as well as future directions.

\section{Compliance with Ethics Guidelines}

This article is based on previously conducted studies and does not involve any new studies of human or animal subjects performed by any of the authors.

\section{Study Selection Criteria}

Our review focused on the literature surrounding multiple sleep features and the relationship with measures/indicators of type 2 diabetes mellitus. Specifically, we reviewed the literature for human, adult ( $\geq 18$ years) populations and excluded animal studies or studies not published in the English language. We selected studies using a comprehensive search of the electronic databases, PubMed and MEDLINE. The search terms entered into these electronic databases in May 2015 included: type 2 diabetes mellitus, insulin resistance, insulin sensitivity, glycemic control, glucose control and sleep duration, sleep quality, circadian misalignment, circadian preference, chronotype and napping. The search highlighted a total of 83 studies of which there were 6 case-control, 41 cross-sectional, 15 prospective cohort and 21 experimental studies.

\section{Sleep Quantity, Insulin Resistance and Diabetes}

Sleep curtailment is now widespread, usually to accommodate busy lifestyles within contemporary society, mirroring the increases in global diabetes mellitus [2]. The use of modern technology is also increasingly 
Table 1 The advantages and limitations of the various methods used to measure sleep

\begin{tabular}{|c|c|c|c|}
\hline Sleep measure & Measure & Advantages & Limitations \\
\hline \multirow[t]{6}{*}{$\begin{array}{l}\text { Polysomnography } \\
\text { (PSG) }\end{array}$} & Objective & $\begin{array}{l}\text { Accurate for determining multiple sleep } \\
\text { parameters }\end{array}$ & Expensive \\
\hline & Gold standard & Can be used to diagnose sleep disorders & $\begin{array}{l}\text { Experienced/trained technicians are } \\
\text { needed to score the data }\end{array}$ \\
\hline & Physiological & $\begin{array}{l}\text { Can be combined with other } \\
\text { physiological measures (hormone } \\
\text { sampling under controlled conditions) }\end{array}$ & $\begin{array}{l}\text { May not be able to capture usual sleep } \\
\text { because of equipment and/or } \\
\text { environment (first night effect) }\end{array}$ \\
\hline & & $\begin{array}{l}\text { Determines sleep architecture (sleep } \\
\text { stages and percentages of each stage) }\end{array}$ & Invasive/uncomfortable \\
\hline & $\begin{array}{l}\text { Sleep } \\
\text { architecture } \\
\text { (stages 1, 2, } 3 \\
\text { and REM } \\
\text { sleep) }\end{array}$ & $\begin{array}{l}\text { Measures brain activity as well as other } \\
\text { physiological outcomes (muscle } \\
\text { relaxation, eye movements respiratory } \\
\text { effort and more) }\end{array}$ & $\begin{array}{l}\text { Unsuitable for long-term sleep } \\
\text { assessment; unless portable requires } \\
\text { laboratory attendance }\end{array}$ \\
\hline & & & Inter-/intraobserver variation \\
\hline \multirow[t]{7}{*}{ Actigraphy (wrist) } & $\begin{array}{l}\text { Objective } \\
\text { estimate }\end{array}$ & Objective measure of sleep-wake timings & $\begin{array}{l}\text { Cannot determine sleep architecture } \\
\text { (sleep stages) }\end{array}$ \\
\hline & & $\begin{array}{l}\text { Can be used in the individual's natural } \\
\text { environment in free-living conditions }\end{array}$ & $\begin{array}{l}\text { Provides an estimate of sleep-wake } \\
\text { timings }\end{array}$ \\
\hline & Worn on wrist & $\begin{array}{l}\text { Some devices have been validated for } \\
\text { sleep duration against PSG }\end{array}$ & $\begin{array}{l}\text { Some devices are not waterproof and } \\
\text { will not capture information upon } \\
\text { removal }\end{array}$ \\
\hline & $\begin{array}{l}\text { Based on } \\
\text { movement }\end{array}$ & $\begin{array}{l}\text { Ability to collect data over prolonged } \\
\text { periods of time (up to } 3 \text { consecutive } \\
\text { months) }\end{array}$ & $\begin{array}{l}\text { Absence of physiological measures to } \\
\text { determine sleep }\end{array}$ \\
\hline & & Cost-effective alternative to PSG & $\begin{array}{l}\text { Requires concurrent sleep diary and } \\
\text { minimum wear time }\end{array}$ \\
\hline & & Noninvasive & $\begin{array}{l}\text { May over estimate sleep during periods } \\
\text { of inactivity }\end{array}$ \\
\hline & & & $\begin{array}{l}\text { Multiple software and cut points for } \\
\text { analysis }\end{array}$ \\
\hline \multirow[t]{2}{*}{ Actiheart } & $\begin{array}{l}\text { Objective } \\
\text { estimate }\end{array}$ & Objective & Not validated against PSG for sleep \\
\hline & & $\begin{array}{l}\text { Additional physiological measures are } \\
\text { obtained (heart rate) for sleep } \\
\text { determination }\end{array}$ & $\begin{array}{l}\text { Loss of signal if skin contact is poor or } \\
\text { the ECG pads become loose/removed }\end{array}$ \\
\hline
\end{tabular}


Table 1 continued

\begin{tabular}{|c|c|c|c|}
\hline Sleep measure & Measure & Advantages & Limitations \\
\hline & Physiological & $\begin{array}{l}\text { Can be used in free-living conditions and } \\
\text { natural environments }\end{array}$ & $\begin{array}{l}\text { Does not have the ability to determine } \\
\text { sleep architecture (sleep staging) }\end{array}$ \\
\hline & & $\begin{array}{l}\text { Can collect data over prolonged periods } \\
\text { of time }\end{array}$ & $\begin{array}{l}\text { Accurate accompanying sleep diary is } \\
\text { usually required }\end{array}$ \\
\hline & & Noninvasive & $\begin{array}{l}\text { May overestimate sleep during periods } \\
\text { of inactivity }\end{array}$ \\
\hline & & & $\begin{array}{l}\text { Can be uncomfortable and/or result in } \\
\text { skin irritation where ECG electrodes } \\
\text { are placed }\end{array}$ \\
\hline & & & Expensive \\
\hline \multirow[t]{7}{*}{$\begin{array}{l}\text { Self-reported } \\
\text { questionnaires }\end{array}$} & Subjective & Can be administered to large populations & $\begin{array}{l}\text { Subject to a number of biases (recall, } \\
\text { social desirability) }\end{array}$ \\
\hline & & Quick/easy to administer & Variable response rates \\
\hline & & Cost-effective & Subjective \\
\hline & & $\begin{array}{l}\text { Some are validated in different age groups } \\
\text { to investigate different age-appropriate } \\
\text { sleep problems (pain in the elderly, } \\
\text { bedroom sharing in children) }\end{array}$ & Inaccurate for detecting sleep disorders \\
\hline & & Less labor intensive compared to PSG & May be subject to missing data \\
\hline & & $\begin{array}{l}\text { Some are validated for sleep duration } \\
\text { against objective measures of sleep }\end{array}$ & $\begin{array}{l}\text { May result in time in bed being } \\
\text { reported rather than total sleep time }\end{array}$ \\
\hline & & $\begin{array}{l}\text { Can help to ascertain information about } \\
\text { multiple sleep parameters and other } \\
\text { related factors }\end{array}$ & $\begin{array}{l}\text { Information collected may not be } \\
\text { accurate and some only ask one } \\
\text { question }\end{array}$ \\
\hline \multirow{5}{*}{$\begin{array}{l}\text { Parental } \\
\text { questionnaire }\end{array}$} & Subjective & Inexpensive & Subjective \\
\hline & & Administration is quick & $\begin{array}{l}\text { Subject to a number of biases (recall, } \\
\text { social desirability) }\end{array}$ \\
\hline & & Immediate output & May have missing data \\
\hline & & $\begin{array}{l}\text { Permits data collection in large samples } \\
\text { relating to pediatric sleep information }\end{array}$ & $\begin{array}{l}\text { Likely to be inaccurate for older } \\
\text { children and adolescents (parents } \\
\text { may be unaware of night awakenings } \\
\text { and/or other sleep features) }\end{array}$ \\
\hline & & Less labor intensive compared to PSG & Variable response rates \\
\hline
\end{tabular}


Table 1 continued

\begin{tabular}{|c|c|c|c|}
\hline Sleep measure & Measure & Advantages & Limitations \\
\hline & & & $\begin{array}{l}\text { May result in time in bed being } \\
\text { reported rather than total sleep time, } \\
\text { thus overestimating sleep causing } \\
\text { inaccuracies }\end{array}$ \\
\hline \multirow[t]{6}{*}{ Sleep/time diary } & Subjective & Obtains prospective sleep-wake data & Completion is tedious \\
\hline & & $\begin{array}{l}\text { Provides additional information about } \\
\text { other sleep features (time in bed, sleep } \\
\text { duration, night awakenings, napping, } \\
\text { sleep quality) }\end{array}$ & $\begin{array}{l}\text { Response rates may be low or diaries } \\
\text { may be only partially completed } \\
\text { (missing data) }\end{array}$ \\
\hline & & Inexpensive & Subjective \\
\hline & & Permits data collection in large samples & Labor intensive for the participant \\
\hline & & Less labor intensive compared to PSG & $\begin{array}{l}\text { Requires participants to be motivated } \\
\text { to complete }\end{array}$ \\
\hline & & Swift administration & $\begin{array}{l}\text { Subject to inaccuracies/biases (recall, } \\
\text { social desirability) }\end{array}$ \\
\hline
\end{tabular}

$R E M$ rapid eye movement, $P S G$ polysomnography

intruding into sleep time. The Sleep Heart Health Study is an early study that observed, in a large sample of US men and women, that short self-reported sleep duration $(\leq 5 \mathrm{~h}$ per night) was associated with a $251 \%$ increased odds ratio (OR) of T2DM [3]. Furthermore, compared to those with sleep duration of $7-8 \mathrm{~h}$, those with sleep duration of $\geq 9 \mathrm{~h}$ had a $79 \%$ increased odds of T2DM and an $88 \%$ increased odds of pre-diabetes. This early study adjusted for age, gender, ethnicity, the apnea hypopnea index (AHI), study recruitment site, and waist circumference as potential confounders, although data on primary drivers of diabetes (physical activity, dietary habits, family history) were not included in the analyses [3]. Further evidence has resulted from a small number of case-control studies that are highlighted in Table 2.

\section{Cross-Sectional Studies}

The cross-sectional evidence linking sleep duration to T2DM has continued to accumulate (Table 3). The majority of studies have indicated an increased possibility of various diabetes outcomes with differing definitions of short sleep duration [4-17]. Understanding pre-diabetes provides a key insight into the development of T2DM. Chaput and colleagues performed an oral glucose tolerance test in 740 participants, aged 21-64 years without a known diabetes diagnosis. Sleep duration (h) was self-reported from only one question, inserted into a self-administered physical activity questionnaire. After adjustment for major confounders such as physical activity and energy intake (self-reported) along with age, marital and employment status, education, 


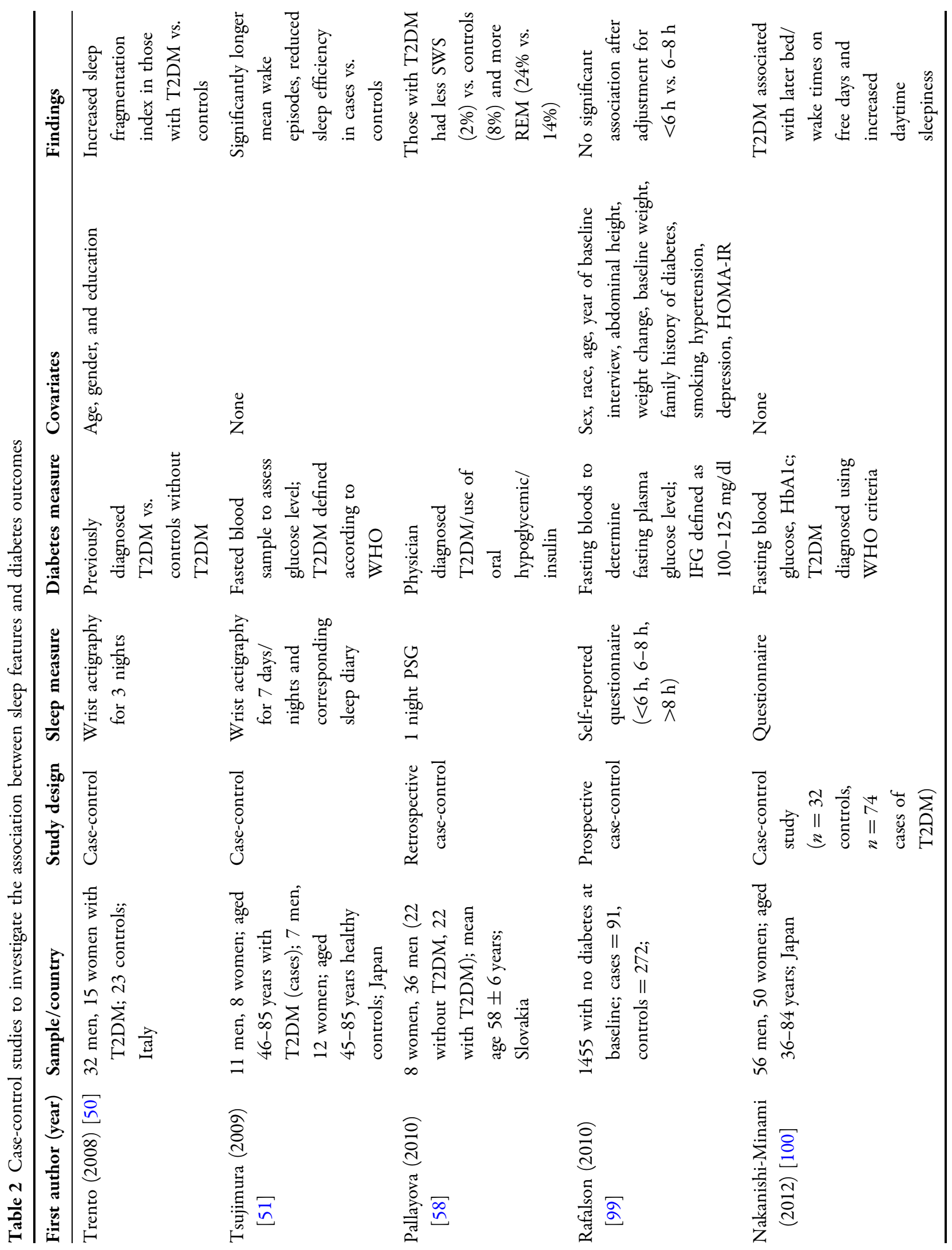




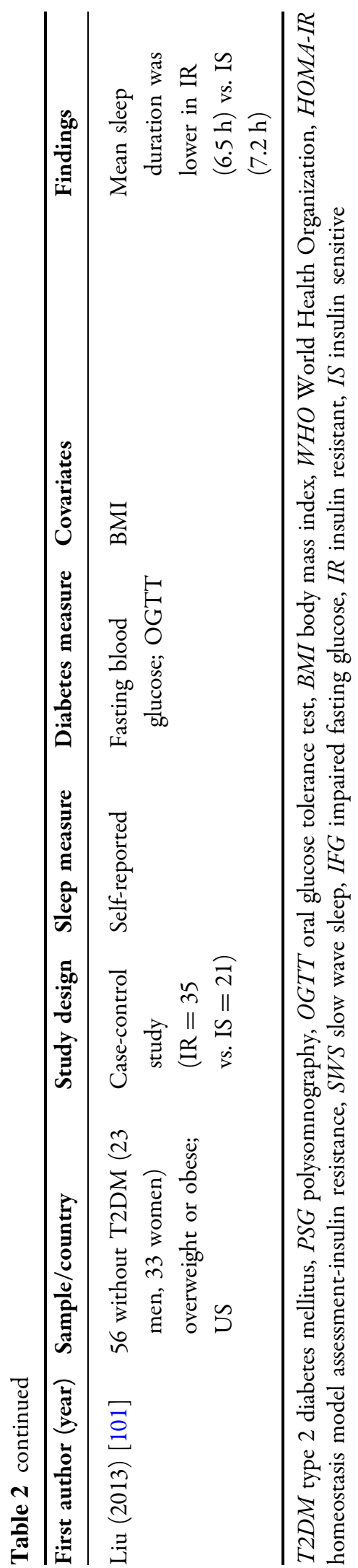

household income, alcohol and coffee consumption, hypertension and heart disease, the OR for insulin resistance, determined using the homeostasis model assessment (HOMA-IR), for men and women reporting short sleep duration (5-6 h per night) was 2.27 and 1.82, respectively (both $p<0.05$ ). Long sleep duration (9-10 h per night) was also significantly associated with pre-diabetes in both men and women, but with smaller ORs of 1.51 and 1.67, respectively [4]. While this study included many potential confounders and benefited from a relatively large sample, a major concern is the limited subjective sleep information.

To overcome the issue of subjective sleep reports, Vgontzas and colleagues employed polysomnography (PSG; the gold-standard sleep measure) to investigate the relationship between sleep and T2DM. A fasted blood glucose sample was obtained the morning after 1 night's PSG had been conducted to screen participants for sleep-disordered breathing (SDB), known to be closely associated with T2DM. Severe short sleep duration $(\leq 5 \mathrm{~h})$ was significantly associated with a 2.95 times odds of T2DM [18]. While the authors adjusted for a range of potential confounders including age, race, sex, BMI, sampling weight, smoking, alcohol, depression and SDB, the two major lifestyle drivers (diet and physical activity) and family history of diabetes were unaccounted for. Investigating sleep in those susceptible to diabetes has provided key information regarding the sleep-diabetes relationship. Darukhanavala et al. studied 47 at-risk participants with a family history of diabetes but who were otherwise healthy and monitored them with wrist actigraphy for 14 consecutive days/nights. Insulin sensitivity was determined using HOMA-IR, and a significant linear relationship 
Table 3 Cross-sectional studies investigating the association between sleep features and diabetes outcomes

\begin{tabular}{|c|c|c|c|c|c|}
\hline $\begin{array}{l}\text { First author } \\
\text { (year) }\end{array}$ & Sample/country & Sleep measure & Diabetes measure & Covariates & Findings \\
\hline $\begin{array}{l}\text { Gottlieb (2005) } \\
\text { [3] }\end{array}$ & $\begin{array}{l}722 \text { men; } 764 \\
\text { women; age } \\
53-93 \text { years; } \\
\text { US }\end{array}$ & $\begin{array}{l}\text { Self-reported sleep } \\
\text { duration } \\
\text { (h) from } \\
\text { questionnaire }\end{array}$ & $\begin{array}{l}\text { Fasting blood sample } \\
\text { OGTT; T2DM } \\
\text { and IGT (ADA } \\
\text { and WHO) }\end{array}$ & $\begin{array}{l}\text { Age, gender, } \\
\text { ethnicity, AHI, } \\
\text { study site, waist } \\
\text { circumference }\end{array}$ & $\begin{array}{l}\text { T2DM } \leq 5 \mathrm{~h} \\
\mathrm{HR}=2.51^{*} ; \\
\text { T2DM } \geq 9 \mathrm{~h} \\
\mathrm{HR}=1.79^{*} \\
\mathrm{IGT} \leq 5 \mathrm{~h} \\
\mathrm{HR}=1.33 \\
\mathrm{IGT} \geq 9 \mathrm{~h} \\
\mathrm{HR}=1.88^{*}\end{array}$ \\
\hline $\begin{array}{l}\text { Fiorentini } \\
\qquad(2007) \text { [43] }\end{array}$ & $\begin{array}{l}220 \text { (men and } \\
\text { women); no age } \\
\text { range provided; } \\
\text { Italy }\end{array}$ & $\begin{array}{c}\text { Self-reported sleep } \\
\text { quality (PSQI) }\end{array}$ & $\begin{array}{l}\text { Diagnosed T2DM } \\
\text { (ADA) }\end{array}$ & None & $\begin{array}{l}\text { Prevalence of } \\
\text { T2DM was } \\
19.4 \% \text { in 'poor } \\
\text { sleepers' versus } \\
8.8 \% \text { in 'good } \\
\text { sleepers' } \\
(p<0.0001)\end{array}$ \\
\hline $\begin{array}{l}\text { Knutson } \\
\text { (2006) [41] }\end{array}$ & $\begin{array}{l}161 \text { ( } 42 \text { men; } 119 \\
\text { women); } \\
\text { African } \\
\text { American; } \\
\text { mean age } \\
57 \text { years; US }\end{array}$ & $\begin{array}{l}\text { Sleep quality and } \\
\text { sleep duration } \\
\text { from } \\
\text { self-reported } \\
\text { PSQI; } \\
\text { additional } \\
\text { question on } \\
\text { preferred sleep } \\
\text { duration to } \\
\text { calculate } \\
\text { perceived sleep } \\
\text { debt }\end{array}$ & $\begin{array}{l}\text { Diagnosed T2DM } \\
\text { patients; measure } \\
\text { of glycemic control } \\
\text { determined using } \\
\text { HbAlc }\end{array}$ & & $\begin{array}{l}3 \mathrm{~h} \text { sleep debt } \\
\mathrm{p} / \text { night } \\
\text { associated with } \\
1.1 \% \text { significant } \\
\text { increase in } \\
\text { HbAlc }\end{array}$ \\
\hline $\begin{array}{l}\text { Chaput (2007) } \\
\text { [4] }\end{array}$ & $\begin{array}{l}323 \text { men, } 417 \\
\text { women; } \\
\text { 21-64 years; } \\
\text { Canada }\end{array}$ & $\begin{array}{l}\text { Self-reported sleep } \\
\text { duration (one } \\
\text { question) }\end{array}$ & $\begin{array}{l}\text { IGT and T2DM } \\
\text { (ADA/WHO) } \\
\text { from fasting bloods } \\
\text { and OGTT }\end{array}$ & $\begin{array}{l}\text { Age, marital status, } \\
\text { employment } \\
\text { status, } \\
\text { education, } \\
\text { income, physical } \\
\text { activity, alcohol, } \\
\text { caffeine, energy } \\
\text { intake, } \\
\text { hypertension, } \\
\text { heart disease, } \\
\text { WC/BMI/BF\% }\end{array}$ & $\begin{array}{l}\text { Men: } 5-6 \text { h sleep } \\
\text { OR } 2.27^{*}, \\
\text { 9-10 h OR } \\
\text { 1.51*; women: } \\
\text { 5-6 h OR } 1.82^{*} \text {, } \\
\text { 9-10 h OR } \\
\text { 1.67*; both } \\
\text { genders 5-6 h } \\
\text { OR } 2.09^{*} \text {, } \\
\text { 9-10 h OR } \\
1.58^{*}\end{array}$ \\
\hline
\end{tabular}


Table 3 continued

\begin{tabular}{|c|c|c|c|c|c|}
\hline $\begin{array}{l}\text { First author } \\
\text { (year) }\end{array}$ & Sample/country & Sleep measure & Diabetes measure & Covariates & Findings \\
\hline $\begin{array}{l}\text { Tuomilehto } \\
\text { (2008) [5] }\end{array}$ & $\begin{array}{l}1336 \text { men, } 1434 \\
\text { women aged } \\
45-74 \text { years; } \\
\text { Finland }\end{array}$ & Questionnaire & OGTT & $\begin{array}{l}\text { Age, BMI, } \\
\text { medication(s), } \\
\text { possible OSA, } \\
\text { smoking, } \\
\text { physical activity }\end{array}$ & $\begin{array}{l}\text { Women with } \\
\text { diagnosed } \\
\text { T2DM: }<6 \mathrm{~h} \\
\text { OR } 2.55^{*},>8 \mathrm{~h} \\
\text { OR } 1.76^{*} ; \\
\text { diagnosed } \\
\text { T2DM or } \\
\text { screened T2DM } \\
>8 \text { h OR } 1.71^{*} \\
\text { no association } \\
\text { for men }\end{array}$ \\
\hline $\begin{array}{l}\text { Suarez }(2008) \\
\quad[54]\end{array}$ & $\begin{array}{l}115 \text { men, } 95 \\
\text { women; aged } \\
18-65 \text { years; } \\
\text { US }\end{array}$ & PSQI & $\begin{array}{l}\text { Fasted blood sample } \\
\text { to assess insulin } \\
\text { and glucose; } \\
\text { HOMA-IR } \\
\text { calculated }\end{array}$ & & 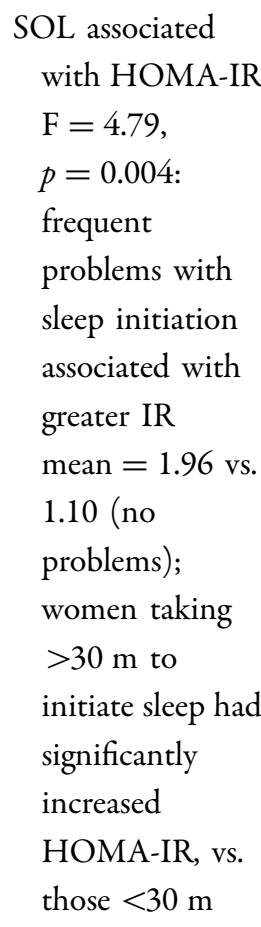 \\
\hline $\begin{array}{l}\text { Cunha (2008) } \\
\text { [44] }\end{array}$ & $\begin{array}{l}50 \text { diabetes } \\
\text { patients; Brazil }\end{array}$ & PSQI & $\begin{array}{l}\text { Previous physician } \\
\text { diagnosed T2DM }\end{array}$ & None & $\begin{array}{l}\text { HbAlc }>7 \%, \\
33.3 \% \text { had poor } \\
\text { sleep quality }\end{array}$ \\
\hline
\end{tabular}


Table 3 continued

\begin{tabular}{|c|c|c|c|c|c|}
\hline $\begin{array}{l}\text { First author } \\
\text { (year) }\end{array}$ & Sample/country & Sleep measure & Diabetes measure & Covariates & Findings \\
\hline $\begin{array}{l}\text { Vgontzas } \\
\text { (2009) [18] }\end{array}$ & $\begin{array}{l}1714(48 \% \text { men }) \\
\text { mean age } \\
49 \pm 14 \text { years; } \\
\text { US }\end{array}$ & $\begin{array}{l}\text { One night PSG } \\
\text { and } \\
\text { questionnaire } \\
\text { with } 3 \text { groups: } \\
>6 \mathrm{~h} \text { (normal), } \\
5-6 \mathrm{~h} \\
\text { (moderately } \\
\text { short), } \leq 5 \mathrm{~h} \\
\text { (severely short) }\end{array}$ & $\begin{array}{l}\text { T2DM diagnosis and } \\
\text { treatment or } \\
\text { fasting blood } \\
\text { glucose }>126 \mathrm{mg} / \\
\text { dl on the morning } \\
\text { after sleep study }\end{array}$ & $\begin{array}{l}\text { Age, race, sex, } \\
\text { BMI, sampling } \\
\text { weight, smoking, } \\
\text { alcohol, } \\
\text { depression, } \\
\text { sleep-disordered } \\
\text { breathing }\end{array}$ & $\begin{array}{l}\text { T2DM associated } \\
\text { with insomnia } \\
(<5 \mathrm{~h}) \text { OR } \\
2.95^{*}\end{array}$ \\
\hline $\operatorname{Kim}(2009)[6]$ & $\begin{array}{l}1652 \text { men, aged } \\
20-60 \text { years } \\
\text { with central } \\
\text { adiposity; } \\
\text { Korea }\end{array}$ & $\begin{array}{l}\text { Self-reported } \\
\text { from } \\
\text { questionnaire }\end{array}$ & $\begin{array}{l}\text { T2DM: previous } \\
\text { physician } \\
\text { diagnosis/use of } \\
\text { diabetes } \\
\text { medication/fasting } \\
\text { blood glucose } \\
\geq 7.0 \mathrm{mmol} / 1\end{array}$ & $\begin{array}{l}\text { Age, smoking, } \\
\text { alcohol, physical } \\
\text { activity, } \\
\text { education, } \\
\text { income, } \\
\text { residential area, } \\
\text { hypertension, } \\
\text { obesity, } \\
\text { abdominal } \\
\text { obesity, high } \\
\text { triglycerides, low } \\
\text { HDL-C, high } \\
\text { cholesterol }\end{array}$ & $\begin{array}{l}\text { OR } 2.40^{*} \text { for } \\
\text { T2DM if } \leq 5 \mathrm{~h} \\
\text { without } \\
\text { abdominal } \\
\text { obesity }\end{array}$ \\
\hline $\begin{array}{l}\text { Chao (2011) } \\
\text { [7] }\end{array}$ & $\begin{array}{l}3470 \text { adults; } \\
\text { Taiwan }\end{array}$ & $\begin{array}{l}\text { Self-reported } \\
\text { questionnaire } \\
(<6 \mathrm{~h}, \\
6-8.49 \mathrm{~h}, \\
\geq 8.5 \mathrm{~h})\end{array}$ & $\begin{array}{l}\text { Pre-diabetes and } \\
\text { T2DM diagnosed } \\
\text { from either fasted } \\
\text { blood sample or } \\
\text { OGTT }\end{array}$ & $\begin{array}{l}\text { Age, sex, smoking, } \\
\text { alcohol, caffeine, } \\
\text { physical activity, } \\
\text { family history of } \\
\text { diabetes, obesity }\end{array}$ & $\begin{array}{l}\text { Short sleep and } \\
\text { T2DM OR } \\
\text { 1.55*; long sleep } \\
\text { and T2DM OR } \\
\text { 2.83; no } \\
\text { association with } \\
\text { pre-diabetes }\end{array}$ \\
\hline $\begin{array}{l}\text { Knutson } \\
\qquad(2011) \text { [39] }\end{array}$ & $\begin{array}{l}115 \text { without } \\
\text { T2DM, } 40 \\
\text { with T2DM; } \\
\text { 18-30 years; } \\
\text { US }\end{array}$ & $\begin{array}{l}\text { Wrist actigraphy } \\
\text { for } 6 \text { days/ } \\
\text { nights and } \\
\text { questionnaires } \\
\text { (PSQI, Berlin) }\end{array}$ & $\begin{array}{l}\text { Fasting bloods to } \\
\text { measure insulin/ } \\
\text { glucose and } \\
\text { calculate } \\
\text { HOMA-IR }\end{array}$ & $\begin{array}{l}\text { Age, race, sex, } \\
\text { BMI, education, } \\
\text { income }\end{array}$ & $\begin{array}{l}\text { Sleep } \\
\text { fragmentation } \\
\text { and positively } \\
\text { associated with } \\
\text { insulin and } \\
\text { HOMA-IR }\end{array}$ \\
\hline $\begin{array}{l}\text { Luyster (2011) } \\
{[45]}\end{array}$ & $\begin{array}{l}300 \text { with T2DM; } \\
\text { mean age } \\
64 \text { years; US }\end{array}$ & PSQI & $\begin{array}{l}\text { Physician diagnosis of } \\
\text { T2DM for at least } \\
\text { ly and taking oral } \\
\text { medication }\end{array}$ & & $\begin{array}{l}55 \% \text { of the sample } \\
\text { had poor sleep } \\
\text { quality }\end{array}$ \\
\hline
\end{tabular}


Table 3 continued

\begin{tabular}{|c|c|c|c|c|c|}
\hline $\begin{array}{l}\text { First author } \\
\text { (year) }\end{array}$ & Sample/country & Sleep measure & Diabetes measure & Covariates & Findings \\
\hline $\begin{array}{c}\text { Darukhanavala } \\
\text { (2011) [19] }\end{array}$ & $\begin{array}{l}47 \text { healthy } \\
\text { individuals with } \\
\text { parental history } \\
\text { of T2DM ( } 26 \\
\text { women, } 21 \\
\text { men); mean age } \\
26 \text { years; US }\end{array}$ & $\begin{array}{l}14 \text { days/night } \\
\text { wrist actigraphy }\end{array}$ & OGTT, HOMA-IR & $\begin{array}{l}\text { Age, BMI, sex, } \\
\text { familial diabetes } \\
\text { risk, ethnicity, } \\
\text { physical activity }\end{array}$ & $\begin{array}{l}\text { Sleep duration } \\
\text { associated with } \\
\text { insulin } \\
\text { sensitivity } \\
\beta=2.5^{*} \text { and } \\
\text { HOMA-IR } \\
\beta=-0.27\end{array}$ \\
\hline $\begin{array}{l}\text { Tsai }(2012) \\
{[46]}\end{array}$ & $\begin{array}{l}46 \text { with T2DM; } \\
\text { aged } \\
\text { 43-83 years; } \\
\text { Taiwan }\end{array}$ & PSQI & $\begin{array}{l}\text { HbAlc from blood } \\
\text { draw }\end{array}$ & Age, gender, BMI & $\begin{array}{l}\text { OR } 6.83^{*} \text { for sleep } \\
\text { efficiency and } \\
\text { HbAlc; Poor } \\
\text { quality sleep } \\
\text { associated with } \\
\text { worse glycemic } \\
\text { control OR } \\
6.94^{*}\end{array}$ \\
\hline Liu (2011) [8] & $\begin{array}{l}854 \text { men, } 640 \\
\text { women; aged } \\
\text { 20-70 years; } \\
\text { twin cohort; } \\
\text { China }\end{array}$ & $\begin{array}{l}\text { Sleep duration } \\
\text { self-reported } \\
\text { from PSQI }\end{array}$ & $\begin{array}{c}\text { Fasting plasma } \\
\text { glucose and } \\
\text { HOMA-IR }\end{array}$ & $\begin{array}{l}\text { Age, physical } \\
\text { activity, } \\
\text { education, } \\
\text { snoring, sleep } \\
\text { disturbances, } \\
\text { BMI/\%TF }\end{array}$ & $\begin{array}{l}\text { Short sleep } \\
\text { duration }(\leq 7 \mathrm{~h}) \\
\text { associated with } \\
\text { higher } \\
\text { HOMA-IR in } \\
\text { women only }\end{array}$ \\
\hline $\begin{array}{l}\text { St-Onge (2012) } \\
{[55]}\end{array}$ & $\begin{array}{l}305 \text { (122 men, } \\
183 \text { women); } \\
\text { mean age } \\
61 \text { years with } \\
\text { T2DM, } \\
\text { overweight or } \\
\text { obesity; US }\end{array}$ & $\begin{array}{l}\text { Portable PSG in } \\
\text { home } \\
\text { environment }\end{array}$ & $\begin{array}{l}\text { HbAlc, and glucose } \\
\text { from fasting blood } \\
\text { sample }\end{array}$ & $\begin{array}{l}\text { Study site, age, } \\
\text { gender, } \\
\text { ethnicity, WC, } \\
\text { smoking, } \\
\text { alcohol, diabetes } \\
\text { duration, } \\
\text { medication }\end{array}$ & $\begin{array}{l}\text { Sleep efficiency } \\
\text { associated with } \\
\text { fasting plasma } \\
\text { glucose } \\
\beta=-0.53, \\
p=0.041\end{array}$ \\
\hline $\begin{array}{l}\text { Rajendran } \\
\quad(2012)[47]\end{array}$ & $\begin{array}{l}120 \text { with T2DM; } \\
\text { India }\end{array}$ & PSQI & $\begin{array}{c}\text { Fasting, postprandial } \\
\text { blood glucose and } \\
\text { HbAlc measured }\end{array}$ & $\begin{array}{l}\text { Age, sex, } \\
\text { medications, } \\
\text { BMI, HbAlc }\end{array}$ & $\begin{array}{l}\text { Diabetes duration } \\
\text { was negatively } \\
\text { associated with } \\
\text { global PSQI } \\
\mathrm{B}=-0.20, \\
p=0.02\end{array}$ \\
\hline
\end{tabular}


Table 3 continued

\begin{tabular}{|c|c|c|c|c|c|}
\hline $\begin{array}{l}\text { First author } \\
\text { (year) }\end{array}$ & Sample/country & Sleep measure & Diabetes measure & Covariates & Findings \\
\hline $\begin{array}{l}\text { Harada (2012) } \\
\quad[56]\end{array}$ & $\begin{array}{l}275 \text { men; mean } \\
\text { age } 44 \text { years; } \\
\text { Japan }\end{array}$ & $\begin{array}{l}\text { Wrist actigraphy } \\
\text { and } \\
\text { corresponding } \\
\text { sleep diary for } \\
7 \text { days/nights to } \\
\text { determine sleep } \\
\text { duration and } \\
\text { sleep quality }\end{array}$ & $\begin{array}{l}\text { Fasting plasma } \\
\text { glucose }\end{array}$ & $\begin{array}{l}\text { Age, WC, RDI, } \\
\text { ESS, sleep } \\
\text { duration/ } \\
\text { fragmentation }\end{array}$ & $\begin{array}{l}\text { IFG present in } \\
20 \% \text {; sleep } \\
\text { duration nor } \\
\text { sleep quality } \\
\text { (fragmentation) } \\
\text { was associated } \\
\text { with FPG }\end{array}$ \\
\hline $\begin{array}{l}\text { Kachi (2012) } \\
\text { [9] }\end{array}$ & $\begin{array}{l}\text { 20,744 men; aged } \\
\text { 30-64 years; } \\
\text { Japan }\end{array}$ & $\begin{array}{l}\text { Self-reported } \\
\text { (continuous) } \\
\text { then } \\
\text { categorized } \\
\leq 5 \mathrm{~h}, 6 \mathrm{~h}, 7 \mathrm{~h} \\
\text { and } 8 \geq \mathrm{h}\end{array}$ & $\begin{array}{l}\text { Fasting blood glucose } \\
\text { and HbA1c to } \\
\text { determine } \\
\text { undiagnosed } \\
\text { T2DM (JDS) }\end{array}$ & $\begin{array}{l}\text { Age, obesity, } \\
\text { smoking, alcohol } \\
\text { and physical } \\
\text { activity }\end{array}$ & $\begin{array}{l}\text { Untreated T2DM } \\
\begin{array}{l}(3.4 \%) ; \leq 5 \mathrm{~h} \\
\text { sleep associated } \\
\text { with T2DM OR } \\
1.52^{*} ; 8 \geq \mathrm{h} \\
\text { associated with } \\
\text { T2DM OR } \\
1.39^{*}\end{array}\end{array}$ \\
\hline $\begin{array}{l}\text { Hung (2013) } \\
\quad[49]\end{array}$ & $\begin{array}{l}1805 \text { (healthy, } \\
\text { pre-diabetes, } \\
\text { T2DM); } \\
\text { Taiwan }\end{array}$ & PSQI & $\begin{array}{l}\text { Fasting glucose or } \\
\text { OGTT to } \\
\text { determine normal } \\
\text { glucose tolerance } \\
(n=1217), \\
\text { IFG }=118, \\
\text { IGT }=287, \text { IFG } \\
\text { and IGT }=80, \\
\text { T2DM }=103\end{array}$ & $\begin{array}{l}\text { Age, gender, } \\
\text { glycemic status, } \\
\text { sleep duration, } \\
\text { alcohol, } \\
\text { smoking, } \\
\text { physical activity, } \\
\text { BMI, systolic } \\
\text { blood pressure, } \\
\text { HDL, } \\
\text { triglyceride }\end{array}$ & $\begin{array}{l}\text { Poor sleep quality } \\
\text { associated with } \\
\text { FPG } \beta=1.28^{*} \text {, } \\
\text { post-prandial } \\
\text { glucose } \\
\beta=1.07^{*} \text { and } \\
\text { T2DM } \\
\beta=2.27^{*}\end{array}$ \\
\hline $\begin{array}{l}\text { Lou (2012) } \\
\quad[10]\end{array}$ & $\begin{array}{l}16,893 \text { men and } \\
\text { women; aged } \\
18-75 \text { years; } \\
\text { China }\end{array}$ & $\begin{array}{l}\text { Self-reported sleep } \\
\text { quality and } \\
\text { duration }\end{array}$ & $\begin{array}{l}\text { Two fasting blood } \\
\text { samples; T2DM } \\
\text { defined according } \\
\text { to WHO criteria }\end{array}$ & $\begin{array}{l}\text { Age, sex, } \\
\text { education, } \\
\text { occupation, } \\
\text { BMI, family } \\
\text { history of } \\
\text { diabetes, } \\
\text { smoking, } \\
\text { alcohol, } \\
\text { hypertension, } \\
\text { sleep duration/ } \\
\text { quality }\end{array}$ & $\begin{array}{l}\text { Poor sleep quality } \\
\text { associated with } \\
\text { T2DM OR } \\
1.76^{*} \text {; short } \\
\text { sleep } \leq 6 \mathrm{~h} \\
\text { associated with } \\
\text { T2DM OR } \\
1.25^{*}\end{array}$ \\
\hline
\end{tabular}


Table 3 continued

\begin{tabular}{|c|c|c|c|c|c|}
\hline $\begin{array}{l}\text { First author } \\
\text { (year) }\end{array}$ & Sample/country & Sleep measure & Diabetes measure & Covariates & Findings \\
\hline $\begin{array}{l}\text { Ohkuma } \\
\qquad(2013)[11]\end{array}$ & $\begin{array}{l}4870 \text { with } \\
\text { T2DM; aged } \\
\geq 20 \text { years; } \\
\text { Japan }\end{array}$ & $\begin{array}{l}\text { Self-reported sleep } \\
\text { duration }\end{array}$ & HbAlc & $\begin{array}{l}\text { Age, sex, energy } \\
\text { intake, } \\
\text { depressive } \\
\text { symptoms, } \\
\text { duration of } \\
\text { diabetes, } \\
\text { smoking, } \\
\text { alcohol, physical } \\
\text { activity, insulin } \\
\text { use }\end{array}$ & $\begin{array}{l}\text { Short and long } \\
\text { sleep duration } \\
\text { associated with } \\
\text { higher HbAlc }\end{array}$ \\
\hline $\begin{array}{l}\text { Merikanto } \\
\qquad(2013)[12]\end{array}$ & $\begin{array}{l}\text { 4589; aged } \\
\text { 25-74 years; } \\
\text { Finland }\end{array}$ & $\begin{array}{l}\text { Self-reported } \\
\text { chronotype and } \\
\text { sleep duration } \\
\text { (questionnaires) }\end{array}$ & $\begin{array}{l}\text { Fasted blood sample } \\
\text { to determine } \\
\text { glucose and insulin; } \\
\text { OGTT to } \\
\text { determine insulin } \\
\text { sensitivity }\end{array}$ & $\begin{array}{l}\text { Sex, age, } \\
\text { education, civil } \\
\text { status, sleep } \\
\text { duration, } \\
\text { assessment time }\end{array}$ & $\begin{array}{l}\text { Evening } \\
\text { chronotypes had } \\
2.6 \text { increased } \\
\text { risk of T2DM } \\
\text { vs. morning } \\
\text { types; short } \\
\text { sleep ( } \leq 6 \mathrm{~h} \text { ) } \\
\text { associated with } \\
1.6 \text { increased } \\
\text { risk of T2DM; } \\
\text { no association } \\
\text { with insulin } \\
\text { resistance }\end{array}$ \\
\hline $\begin{array}{l}\text { Chasens (2013) } \\
\text { [57] }\end{array}$ & $\begin{array}{l}107 \text { with T2DM; } \\
\text { aged } \\
\text { 31-82 years; } \\
\text { US }\end{array}$ & PSQI and ESS & $\begin{array}{l}\text { Self-reported T2DM } \\
\text { diagnosis; } \\
\text { questionnaire to } \\
\text { assess diabetes care } \\
\text { profile }\end{array}$ & $\begin{array}{l}\text { Sex, age, } \\
\text { education, } \\
\text { marital status, } \\
\text { ESS }\end{array}$ & $\begin{array}{l}\text { Poor sleep quality } \\
\text { associated with } \\
\text { worse diabetes } \\
\text { care profile; } \\
\text { daily } \\
\text { disturbance was } \\
\text { significantly } \\
\text { associated with } \\
\text { increased } \\
\text { diabetes control } \\
\text { problems }\end{array}$ \\
\hline
\end{tabular}


Table 3 continued

\begin{tabular}{|c|c|c|c|c|c|}
\hline $\begin{array}{l}\text { First author } \\
\text { (year) }\end{array}$ & Sample/country & Sleep measure & Diabetes measure & Covariates & Findings \\
\hline $\begin{array}{l}\text { Najafian (2013) } \\
\quad[13]\end{array}$ & $\begin{array}{l}6123 \text { men, } 6391 \\
\text { women; aged } \\
\text { >19 years; Iran }\end{array}$ & Self-reported & $\begin{array}{l}\text { Fasting blood glucose } \\
\text { and OGTT }\end{array}$ & $\begin{array}{l}\text { Age, sex, WC, } \\
\text { BMI }\end{array}$ & $\begin{array}{l}\text { Men sleeping } \\
\leq 5 \mathrm{~h} \text { had } 35 \% \\
\text { increased risk of } \\
\text { T2DM/IGT } \\
\text { and women had } \\
54 \% \text { increased } \\
\text { risk and } \\
<60 \text { years had } \\
34 \% \text { increased } \\
\text { risk }\end{array}$ \\
\hline $\begin{array}{l}\text { Reutrakul } \\
\qquad(2013) \text { [63] }\end{array}$ & $\begin{array}{l}194 \text { ( } 135 \text { women }) \\
\text { with T2DM; } \\
\text { mean age } \\
58 \text { years; US }\end{array}$ & PSQI & $\begin{array}{l}\text { HbAlc from medical } \\
\text { records }\end{array}$ & $\begin{array}{l}\text { Age, sex, race, } \\
\text { BMI, insulin } \\
\text { use, depressed } \\
\text { mood, diabetes } \\
\text { complications, } \\
\text { perceived sleep } \\
\text { debt }\end{array}$ & $\begin{array}{l}\text { Later mid-sleep } \\
\text { time positively } \\
\text { associated with } \\
\text { HbAlc level }\end{array}$ \\
\hline $\begin{array}{l}\text { Kim (2013) } \\
\quad[14]\end{array}$ & $\begin{array}{l}\text { 2134 T2DM } \\
\text { (1065 men, } \\
\text { 1,069 women); } \\
\text { aged >20 years; } \\
\text { Korea }\end{array}$ & Self-reported & $\begin{array}{l}\text { Fasting blood } \\
\text { glucose; HbAlc; } \\
\text { HOMA-IR }\end{array}$ & $\begin{array}{l}\text { Study year, age, } \\
\text { sex, } \\
\text { socioeconomic } \\
\text { status, } \\
\text { education, } \\
\text { marital status, } \\
\text { residential area, } \\
\text { income, alcohol, } \\
\text { smoking, } \\
\text { physical activity, } \\
\text { hypertension, } \\
\text { BMI, WC, } \\
\text { treatment, } \\
\text { T2DM } \\
\text { duration, calorie } \\
\text { intake }\end{array}$ & $\begin{array}{l}\text { No association } \\
\text { between HbAlc } \\
\text { and sleep } \\
\text { duration after } \\
\text { full adjustment; } \\
\text { highest levels of } \\
\text { HOMA-IR with } \\
<6 \mathrm{~h} \text { and } \geq 9 \mathrm{~h} \\
\text { sleep duration }\end{array}$ \\
\hline $\begin{array}{l}\text { Andersson } \\
\qquad(2013)[40]\end{array}$ & $\begin{array}{l}2816 \text { aged } \\
30-75 \text { years; } \\
\text { Sweden }\end{array}$ & $\begin{array}{l}\text { Self-reported lack } \\
\text { of sleep }\end{array}$ & $\begin{array}{l}\text { OGTT to determine } \\
\text { normal glucose } \\
(n=2314) \text { and } \\
\text { IGT }(n=213)\end{array}$ & $\begin{array}{l}\text { Age, BMI, } \\
\text { smoking, } \\
\text { education, } \\
\text { physical activity }\end{array}$ & $\begin{array}{l}\text { IGT and lack of } \\
\text { sleep OR } 2.3^{*} \\
\text { for men only }\end{array}$ \\
\hline
\end{tabular}


Table 3 continued

\begin{tabular}{|c|c|c|c|c|c|}
\hline $\begin{array}{l}\text { First author } \\
\text { (year) }\end{array}$ & Sample/country & Sleep measure & Diabetes measure & Covariates & Findings \\
\hline $\begin{array}{l}\text { Reutrakul } \\
\qquad(2014)[62]\end{array}$ & & & & & $\begin{array}{l}\text { Late chronotype } \\
\text { associated with } \\
\text { higher HbAlc } \\
\text { levels }\end{array}$ \\
\hline $\begin{array}{l}\text { Inkster (2013) } \\
\quad[102]\end{array}$ & $\begin{array}{l}898 \text { with T2DM } \\
\text { (51\% men); } \\
\text { mean age } \\
68 \text { years; UK }\end{array}$ & $\begin{array}{l}\text { Self-reported } \\
\text { daytime } \\
\text { sleepiness from } \\
\text { ESS }\end{array}$ & $\begin{array}{l}\text { Existing T2DM } \\
\text { diagnosis; history } \\
\text { of severe } \\
\text { hypoglycemia } \\
\text { obtained by } \\
\text { questionnaire }\end{array}$ & $\begin{array}{l}\text { Age, sex, T2DM } \\
\text { duration, } \\
\text { HbA1c\%, BMI, } \\
\text { T2DM } \\
\text { medications, } \\
\text { insulin use }\end{array}$ & $\begin{array}{l}\text { ESS was a } \\
\text { significant } \\
\text { independent } \\
\text { predictor of } \\
\text { severe } \\
\text { hypoglycemia } \\
\beta=0.537^{*}\end{array}$ \\
\hline $\begin{array}{l}\text { Ohkuma } \\
\qquad(2014)[103]\end{array}$ & $\begin{array}{l}4402 \text { with T2DM } \\
(2494 \text { men and } \\
1908 \text { women); } \\
\text { aged } \geq 20 \text { years; } \\
\text { Japan }\end{array}$ & $\begin{array}{l}\text { Self-reported sleep } \\
\text { duration, } \\
\text { including } \\
\text { daytime nap }(s)\end{array}$ & $\begin{array}{l}\text { Fasted bloods to } \\
\text { determine HbAlc, } \\
\text { glucose; } \\
\text { HOMA-IR } \\
\text { calculated in } 3816\end{array}$ & $\begin{array}{l}\text { Age, sex, DM } \\
\text { duration, energy } \\
\text { intake, smoking, } \\
\text { alcohol, physical } \\
\text { activity, } \\
\text { depression, DM } \\
\text { medication, } \\
\text { insulin use, } \\
\text { BMI/WC }\end{array}$ & $\begin{array}{l}\text { After adjustment } \\
\text { for BMI/WC, } \\
\text { no significant } \\
\text { association was } \\
\text { found between } \\
\text { HOMA-IR and } \\
\text { sleep duration }\end{array}$ \\
\hline $\begin{array}{l}\text { Cho (2014) } \\
\text { [48] }\end{array}$ & $\begin{array}{l}614 \text { with T2DM } \\
\text { (381 men, } 233 \\
\text { women); mean } \\
\text { age } 60 \text { years; } \\
\text { Korea }\end{array}$ & $\begin{array}{l}\text { PSQI, ESS, Sleep } \\
\text { Disorders } \\
\text { Questionnaire } \\
\text { Sleep Apnea; } \\
\text { poor sleep } \\
\text { quality used as } \\
\text { outcome }\end{array}$ & OGTT & $\begin{array}{l}\text { Age, sex, sleep } \\
\text { apnea score, } \\
\text { depression, } \\
\text { T2DM duration }\end{array}$ & $\begin{array}{l}\text { No significant } \\
\text { association } \\
\text { between glucose } \\
\text { regulation and } \\
\text { any sleep } \\
\text { variable; T2DM } \\
\text { duration } \\
\text { associated with } \\
\text { significantly } \\
\text { higher PSQI }\end{array}$ \\
\hline $\begin{array}{l}\text { Iwasaki (2013) } \\
\text { [65] }\end{array}$ & $\begin{array}{l}101 \text { men with } \\
\text { T2DM; } \\
\text { 40-65 years; } \\
\text { Japan }\end{array}$ & MEQ, PSQI & $\begin{array}{l}\text { HbA1c from blood } \\
\text { sample }\end{array}$ & $\begin{array}{l}\text { Age, BMI, systolic } \\
\text { blood pressure, } \\
\text { HDL-C, } \\
\text { LDL-C, T2DM } \\
\text { duration, } \\
\text { triglycerides }\end{array}$ & $\begin{array}{l}\text { HbAlc negatively } \\
\text { associated with } \\
\text { chronotype; } \\
\text { HbAlc and } \\
\text { PSQI were } \\
\text { lower in } \\
\text { morning types }\end{array}$ \\
\hline
\end{tabular}


Table 3 continued

\begin{tabular}{|c|c|c|c|c|c|}
\hline $\begin{array}{l}\text { First author } \\
\text { (year) }\end{array}$ & Sample/country & Sleep measure & Diabetes measure & Covariates & Findings \\
\hline $\begin{array}{l}\text { Pyykkonen } \\
\quad(2014) \text { [104] }\end{array}$ & $\begin{array}{l}722 \text { without } \\
\text { T2DM ( } 400 \\
\text { women, } 322 \\
\text { men); Finland }\end{array}$ & $\begin{array}{l}\text { Basic Nordic } \\
\text { Sleep } \\
\text { Questionnaire: } \\
\text { sleep duration, } \\
\text { complaints of } \\
\text { sleep apnea and } \\
\text { insomnia }\end{array}$ & OGTT & $\begin{array}{l}\text { Age, sex, sleep } \\
\text { apnea } \\
\text { complaints, } \\
\text { insomnia, family } \\
\text { history of } \\
\text { T2DM, } \\
\text { smoking, } \\
\text { alcohol, physical } \\
\text { activity, } \\
\text { occupation, } \\
\text { BMI, depressive } \\
\text { symptoms }\end{array}$ & $\begin{array}{l}\text { Long }(\geq 9 \mathrm{~h} \text { ) sleep } \\
\text { duration } \\
\text { associated with } \\
\text { increased insulin } \\
\text { resistance }\end{array}$ \\
\hline $\begin{array}{l}\text { Zheng (2015) } \\
\quad[105]\end{array}$ & $\begin{array}{l}18,121(6412 \text { men } \\
\text { and } 11,709 \\
\text { women); aged } \\
\geq 40 \text { years; } \\
\text { Japan }\end{array}$ & $\begin{array}{l}\text { Self-reported sleep } \\
\text { duration }\end{array}$ & $\begin{array}{l}\text { Fasting plasma } \\
\text { glucose; OGTT } \\
\text { and classified: } \\
\text { normal glucose } \\
(n=9578), \\
\text { impaired glucose } \\
\text { regulation } \\
(n=4318), \\
\text { T2DM }(n=4225)\end{array}$ & $\begin{array}{l}\text { Age, sex, BMI, } \\
\text { snoring, } \\
\text { depressive } \\
\text { symptoms }\end{array}$ & $\begin{array}{l}\text { Long sleep } \\
\text { duration }(>9 \mathrm{~h}) \\
\text { associated with } \\
\text { higher HbAlc, } \\
\text { fasting glucose } \\
\text { and } \\
\text { post-prandial } \\
\text { glucose }\end{array}$ \\
\hline $\begin{array}{l}\text { Osonoi (2014) } \\
\text { [64] }\end{array}$ & $\begin{array}{l}725 \text { with T2DM } \\
\text { (63\% men); } \\
\text { mean age } \\
58 \text { years; Japan }\end{array}$ & $\begin{array}{l}\text { Self-reported } \\
\text { chronotype } \\
\text { from MEQ } \\
\text { (n=117 } \\
\text { morning types; } \\
n=424 \\
\text { neither type; } \\
n=184 \\
\text { evening type) }\end{array}$ & $\begin{array}{l}\text { Fasting blood sample } \\
\text { to determine } \\
\text { HbAlc and glucose }\end{array}$ & $\begin{array}{l}\text { Age, gender, BMI, } \\
\text { PSQI, depressive } \\
\text { symptoms, } \\
\text { energy intake, } \\
\text { smoking, } \\
\text { alcohol, physical } \\
\text { activity }\end{array}$ & $\begin{array}{l}\text { Evening } \\
\text { chronotypes had } \\
\text { significantly } \\
\text { higher mean } \\
\text { fasting glucose } \\
\text { and HbAlc }\end{array}$ \\
\hline
\end{tabular}


Table 3 continued

\begin{tabular}{|c|c|c|c|c|c|}
\hline $\begin{array}{l}\text { First author } \\
\text { (year) }\end{array}$ & Sample/country & Sleep measure & Diabetes measure & Covariates & Findings \\
\hline $\begin{array}{l}\text { Baoying (2014) } \\
\quad[106]\end{array}$ & $\begin{array}{l}7568 \text { (3060 men, } \\
4508 \text { women) } \\
\text { without } \\
\text { T2DM; mean } \\
\text { age } 51 \text { years; } \\
\text { China }\end{array}$ & $\begin{array}{l}\text { Self-reported sleep } \\
\text { duration }\end{array}$ & $\begin{array}{l}\text { OGTT to determine } \\
\text { HOMA-IR }\end{array}$ & $\begin{array}{l}\text { Age, gender, } \\
\text { fasting blood } \\
\text { glucose, } \\
\text { hypertension, } \\
\text { FHD, } \\
\text { dyslipidemia, } \\
\text { smoking, } \\
\text { alcohol, snoring } \\
\text { frequency, } \\
\text { physical activity, } \\
\text { education, BMI, } \\
\text { waist-hip ratio }\end{array}$ & $\begin{array}{l}\text { Longer daytime } \\
\text { nap duration } \\
\text { (>1 h) positively } \\
\text { associated with } \\
\text { HbAlc level } \\
>6 \% \text { OR } 1.26^{*} \\
\text { and insulin } \\
\text { resistance OR } \\
1.69^{*} \text {; long sleep } \\
\text { duration ( }>8 \text { h) } \\
\text { had protective } \\
\text { effect on } \\
\text { HbAlc\% and } \\
\text { insulin } \\
\text { resistance OR } \\
0.57^{*} \text { and OR } \\
0.84^{*}\end{array}$ \\
\hline $\begin{array}{l}\text { Wong }(2015) \\
{[15]}\end{array}$ & $\begin{array}{l}224 \text { without } \\
\text { T2DM ( } 52 \% \\
\text { women); mean } \\
\text { age } 45 \text { years; } \\
\text { US }\end{array}$ & $\begin{array}{l}\text { Self-reported sleep } \\
\text { duration }\end{array}$ & $\begin{array}{l}\text { IVGTT to determine } \\
\text { insulin sensitivity }\end{array}$ & & $\begin{array}{l}\text { Short sleep } \\
\text { duration was } \\
\text { associated with } \\
\text { reduced insulin } \\
\text { sensitivity in } \\
\text { Caucasians and } \\
\text { men }\end{array}$ \\
\hline $\begin{array}{l}\text { Tang }(2014) \\
\quad[16]\end{array}$ & $\begin{array}{l}551 \text { with T2DM; } \\
\text { China }\end{array}$ & $\begin{array}{l}\text { PSQI to } \\
\text { determine sleep } \\
\text { quality and } \\
\text { quantity }\end{array}$ & HbAlc; HOMA-IR & $\begin{array}{l}\text { Gender, age, BMI, } \\
\text { T2DM duration }\end{array}$ & $\begin{array}{l}\text { Short sleep } \\
\text { associated with } \\
\text { poorer glycemic } \\
\text { control; poor } \\
\text { sleep quality } \\
\text { associated with } \\
\text { increased insulin } \\
\text { resistance }\end{array}$ \\
\hline $\begin{array}{l}\text { Byberg (2012) } \\
\text { [69] }\end{array}$ & $\begin{array}{l}\text { 771; mean age } \\
47 \text { years; } \\
\text { Denmark }\end{array}$ & $\begin{array}{l}\text { Self-reported sleep } \\
\text { duration } \\
\text { (including } \\
\text { naps) and sleep } \\
\text { quality }\end{array}$ & OGTT; HOMA-IR & & $\begin{array}{l}2 \% \text { increase in } \\
\text { insulin } \\
\text { sensitivity with } \\
\text { improving sleep } \\
\text { quality }\end{array}$ \\
\hline
\end{tabular}


Table 3 continued

\begin{tabular}{|c|c|c|c|c|c|}
\hline $\begin{array}{l}\text { First author } \\
\text { (year) }\end{array}$ & Sample/country & Sleep measure & Diabetes measure & Covariates & Findings \\
\hline $\begin{array}{l}\text { Zuo (2012) } \\
\quad[17]\end{array}$ & $\begin{array}{l}1124 \text { without } \\
\text { T2DM (45\% } \\
\text { men); mean age } \\
\text { 48-49 years; } \\
\text { China }\end{array}$ & $\begin{array}{l}\text { Self-reported sleep } \\
\text { duration }\end{array}$ & HOMA-IR & & $\begin{array}{l}\text { OR } 3.26^{*} \text { for those } \\
\text { with short sleep } \\
(<7 \mathrm{~h}) \text { and low } \\
\text { physical activity } \\
\text { for insulin } \\
\text { resistance; no } \\
\text { association for } \\
\text { sleep duration } \\
\text { alone }\end{array}$ \\
\hline
\end{tabular}

OGTT oral glucose tolerance test, T2DM type 2 diabetes mellitus, $I G T$ impaired glucose tolerance, $A D A$ American Diabetes Association, $W H O$ World Health Organization, $A H I$ apnea hypopnea index, $O R$ odds ratio, OSA obstructive sleep apnea, $B M I$ body mass index, $W C$ waist circumference, $B F \%$ body fat percent, HOMA-IR homeostasis model assessment-insulin resistance, PSQI Pittsburgh Sleep Quality Index, $P S G$ polysomnography, $H D L-C$ high density lipoprotein-cholesterol, $L D L-C$ low high density lipoprotein-cholesterol, $I F G$ impaired fasting glucose, $F P G$ fasting plasma glucose, $R D I$ respiratory disposition index, ESS Epworth Sleepiness Scale, MEQ morningness-eveningness questionnaire, IVGTT intravenous glucose tolerance test ${ }^{*} p<0.05$

was observed between actigraphy-estimated sleep duration and insulin sensitivity $(\beta=2.5$, $p=0.006)$, after adjustment of major confounders such as physical activity and diabetes family history [19].

Currently, there are no cross-sectional studies that have objectively investigated the sleep-diabetes link while adjusting for the three key drivers of diabetes (energy intake, energy expenditure and diabetes family history). The issue with obtaining data from populations with existing diabetes comorbidities, such as $\mathrm{SDB}$, is that sleep insufficiency may be a result of the condition itself, which may have already driven physiological alterations. Thus, case-control (Table 3) and prospective studies provide key evidence for unraveling this and clarifying temporal associations.

\section{Prospective Studies}

A summary of prospective studies that have examined the sleep-diabetes relationship can be found in Table 4. The Niigata Wellness Study, which prospectively studied 38,987 Japanese participants without diabetes over an 8-year period, found that younger individuals ( $<60$ years old) who reported sleeping $<5.5 \mathrm{~h}$ per night had a $53 \%$ significant increased risk of developing diabetes compared to those that slept for 7-7.5 h per night, after adjustment [20]. Gangwisch and colleagues reported a similar significant risk for incident diabetes with short sleep. Participants, aged 32-86 years $(n=8992)$, recruited into the National Health and Nutrition Examination Survey were examined over a 10-year period. Short sleep duration was defined as $\leq 5 \mathrm{~h}$ and was associated with 1.47 increased odds of incident diabetes [21]. Other prospective data suggest similar effect sizes but vary according to adjustment of confounders and short sleep duration definition [22, 23]. The prospective evidence is, however, less consistent with some reporting a diminished relationship after adjustment for 
BMI [24]. Furthermore, at least one study has reported an increased risk for incident diabetes in long sleepers by more than three-fold [25]. The longitudinal data are less convincing than the cross-sectional evidence with several prospective studies indicating no association between sleep duration and incident diabetes [26-28]. Further prospective data are necessary to determine cause-effect associations, and while the majority of studies benefit from large samples, including both genders and incorporating different ethnic backgrounds, the lack of objective sleep data is a significant limitation [20-30]. Also, the majority of prospective studies assessing sleep duration and diabetes outcomes have focused on incident diabetes, with few investigating sleep with pre-diabetes [29, 30]. Both studies, however, documented similar effect sizes that were statistically significant for short sleep duration (albeit with different definitions and varied adjustments). Uncertainty from prospective data has been somewhat offset by evidence from experimental studies. Experimental sleep manipulation in a controlled laboratory environment has shown a consistent relationship between sleep duration and diabetes outcomes.

\section{Experimental Studies}

Increasing numbers of experimental sleep studies (summarized in Table 5) have explored the association between sleep and diabetes using objective measures to confront the problems associated with subjective sleep. One of the earliest experimental sleep studies to examine the effects of sleep upon glucose metabolism recruited healthy, young, male volunteers $(n=11)$ for 16 consecutive nights of laboratory attendance [31]. Participants spent 3 nights of $8 \mathrm{~h}$ time in bed (TIB) for baseline assessment, followed by 6 nights of sleep restriction with $4 \mathrm{~h}$ TIB and finally 7 nights of sleep recovery with $12 \mathrm{~h}$ TIB. PSG was used to monitor sleep outcomes on the last nights of each of the three conditions and blood sampling performed at the end of the sleep restriction and sleep recovery conditions to assess multiple hormones and carbohydrate metabolism. An intravenous glucose tolerance test (IVGTT) and breakfast meal response were performed to investigate insulin sensitivity and glucose effectiveness. Findings from the study indicated a $40 \%$ reduced glucose clearance after the IVGTT following sleep restriction compared to sleep recovery. The insulin response to glucose was reduced by $30 \%$ after sleep restriction, an early indicator of diabetes. Furthermore, glucose effectiveness, which is independent of insulin, was significantly reduced by $30 \%$ after sleep loss versus sleep recovery [31].

To overcome several issues including the potential confounding effect of the presence of diabetes-related comorbidities as well as laboratory attendance and extreme sleep restriction, Zielinski et al. studied 40 healthy individuals (aged 50-70 years) utilizing continuous wrist actigraphy monitoring for 10 weeks [32]. Two weeks of baseline sleep was acquired for determination of habitual sleep habits/duration, and volunteers were subsequently randomized into one of two conditions: (1) 90-min TIB reduction, compared to the pre-determined baseline median TIB, with fixed bedtimes for 8 consecutive weeks $(n=22)$, or (2) control condition with fixed bedtimes, matching the baseline TIB $(n=18)$. An oral glucose tolerance test was conducted to determine glucose and glucose tolerance; insulin sensitivity was assessed using the quantitative insulin-sensitivity check index. The results were not consistent with the findings from 


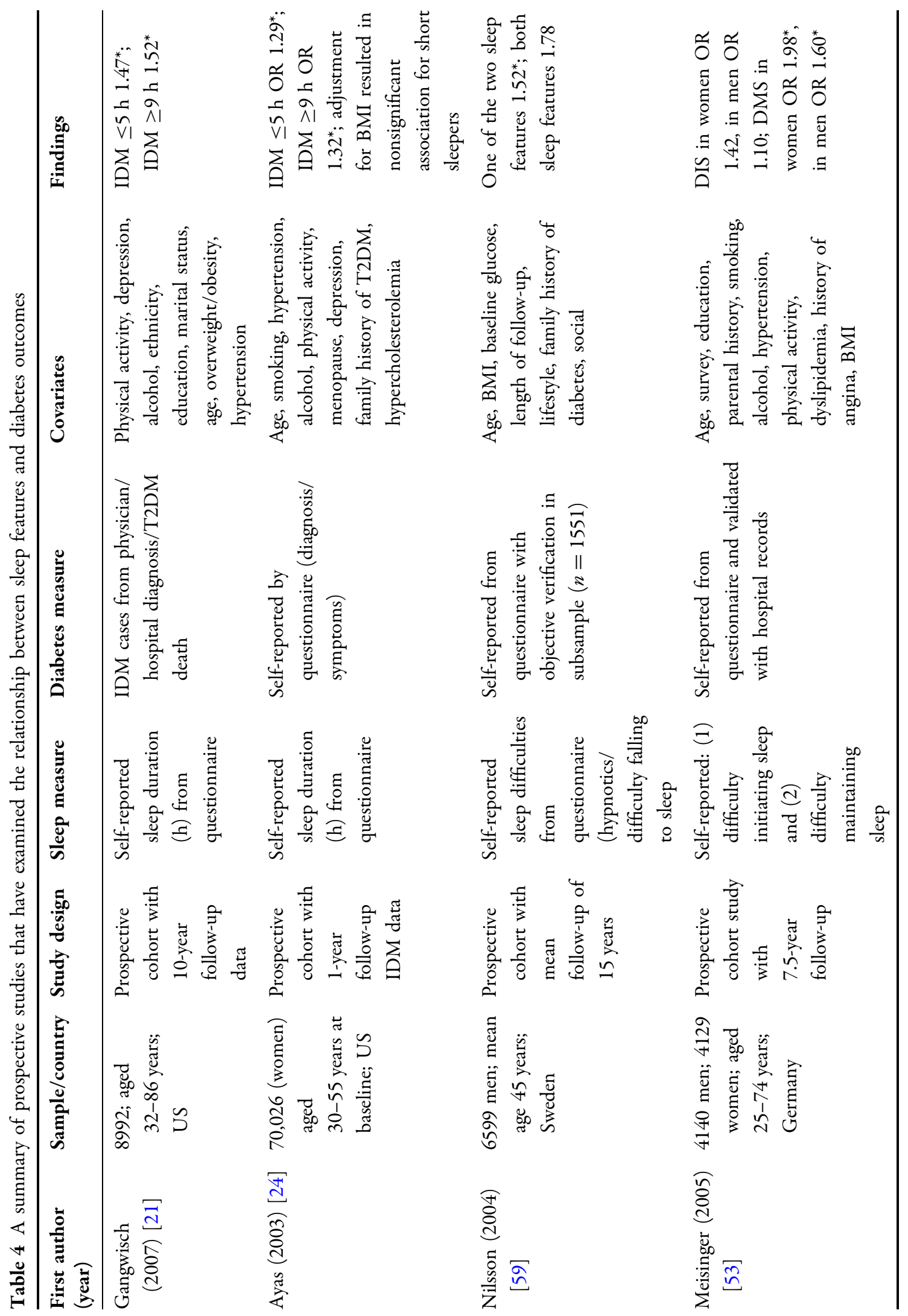




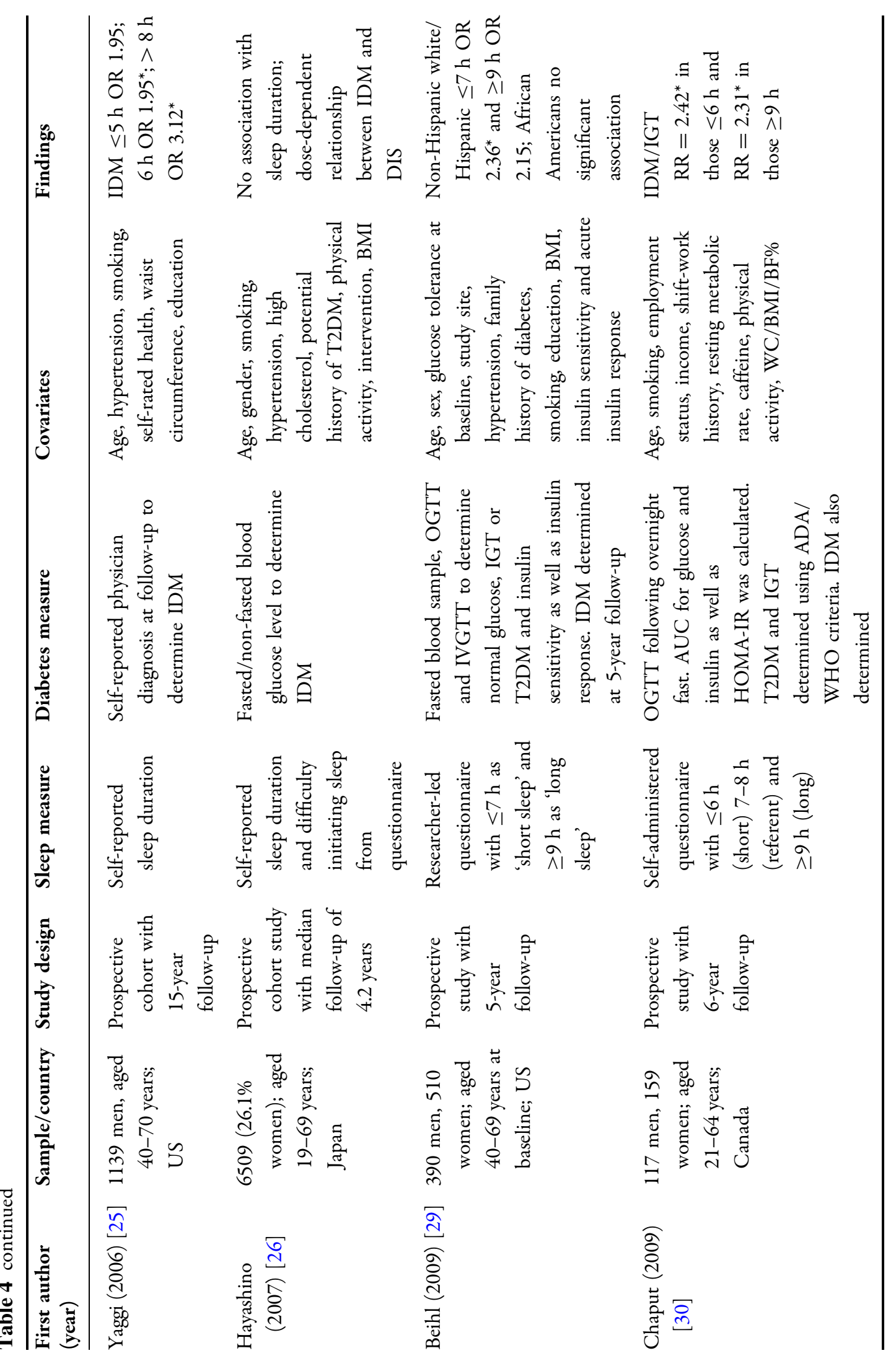




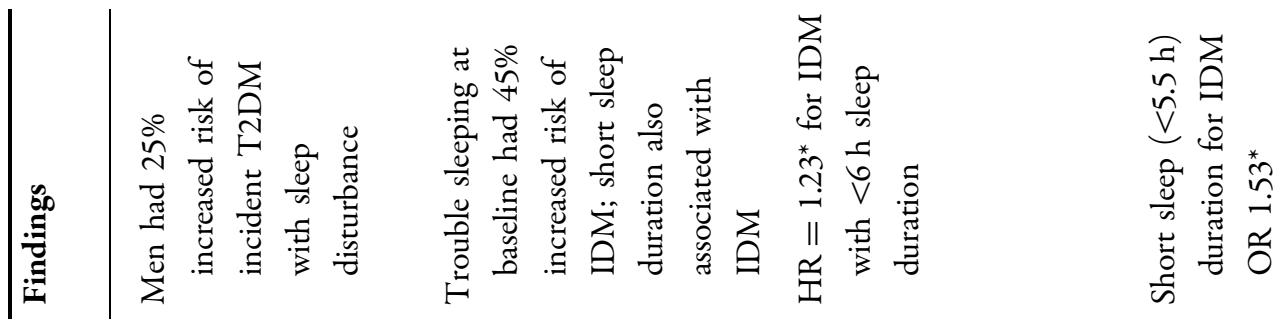

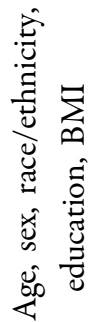

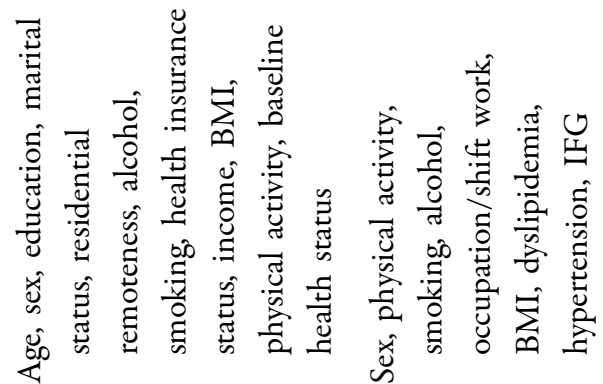

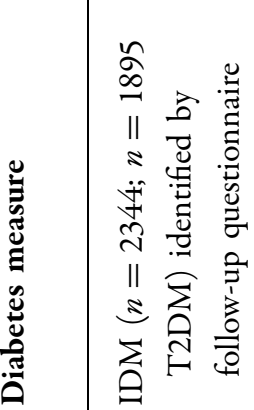

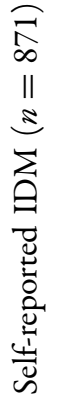

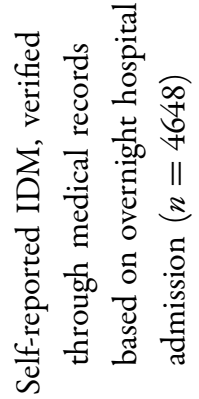

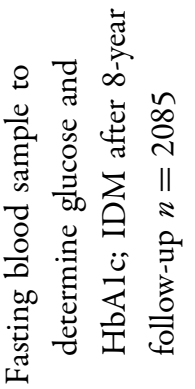

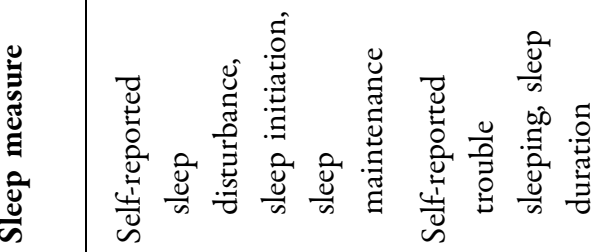

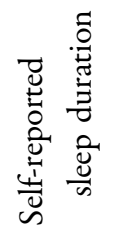

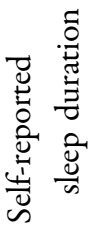

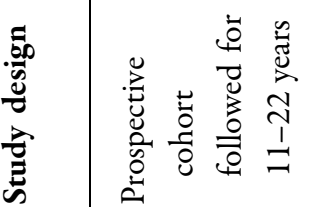

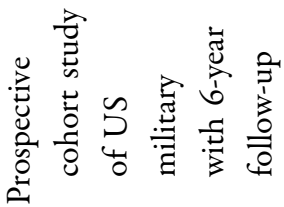

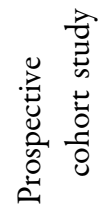

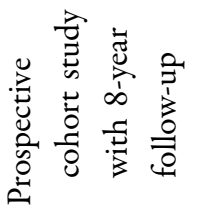

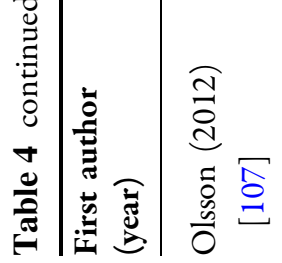

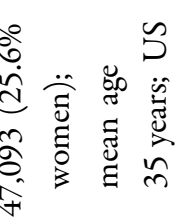

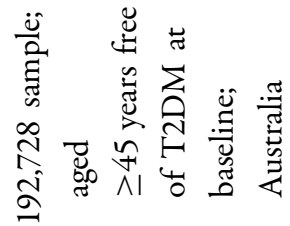

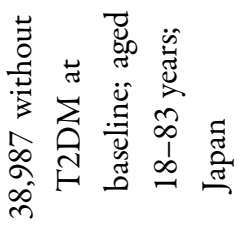

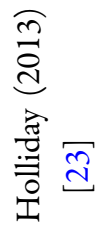

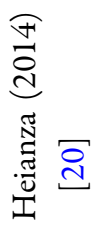




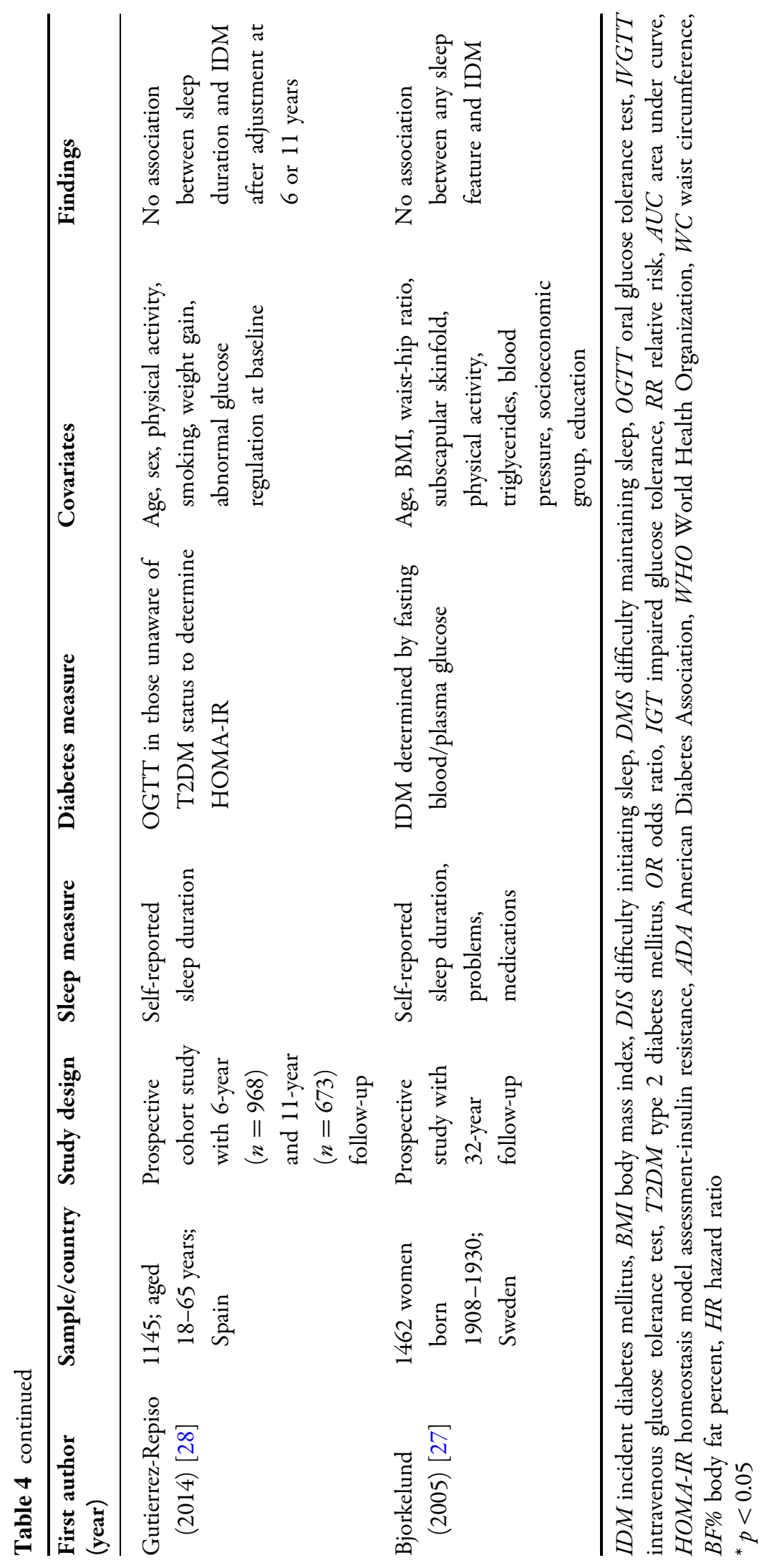




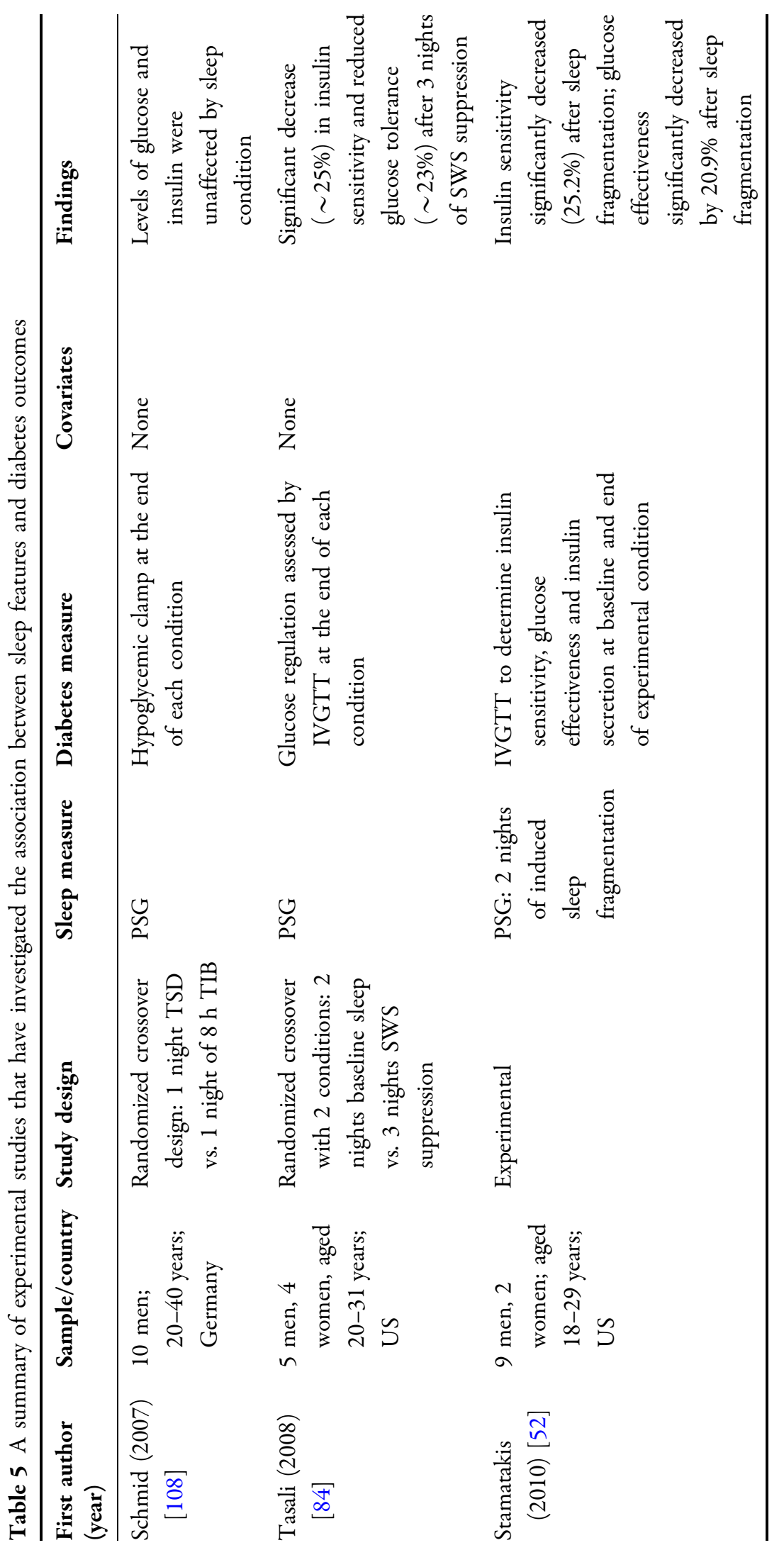




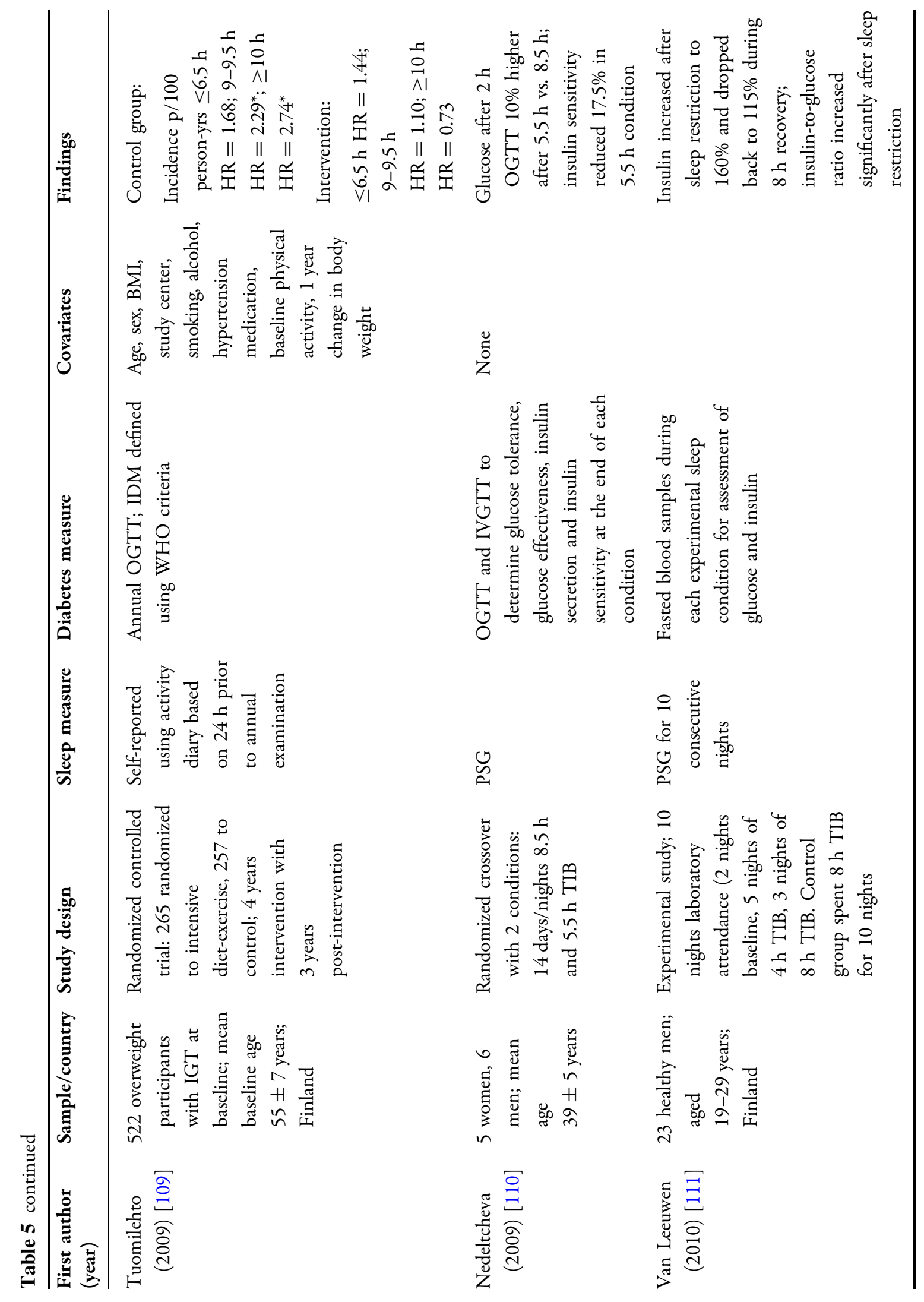




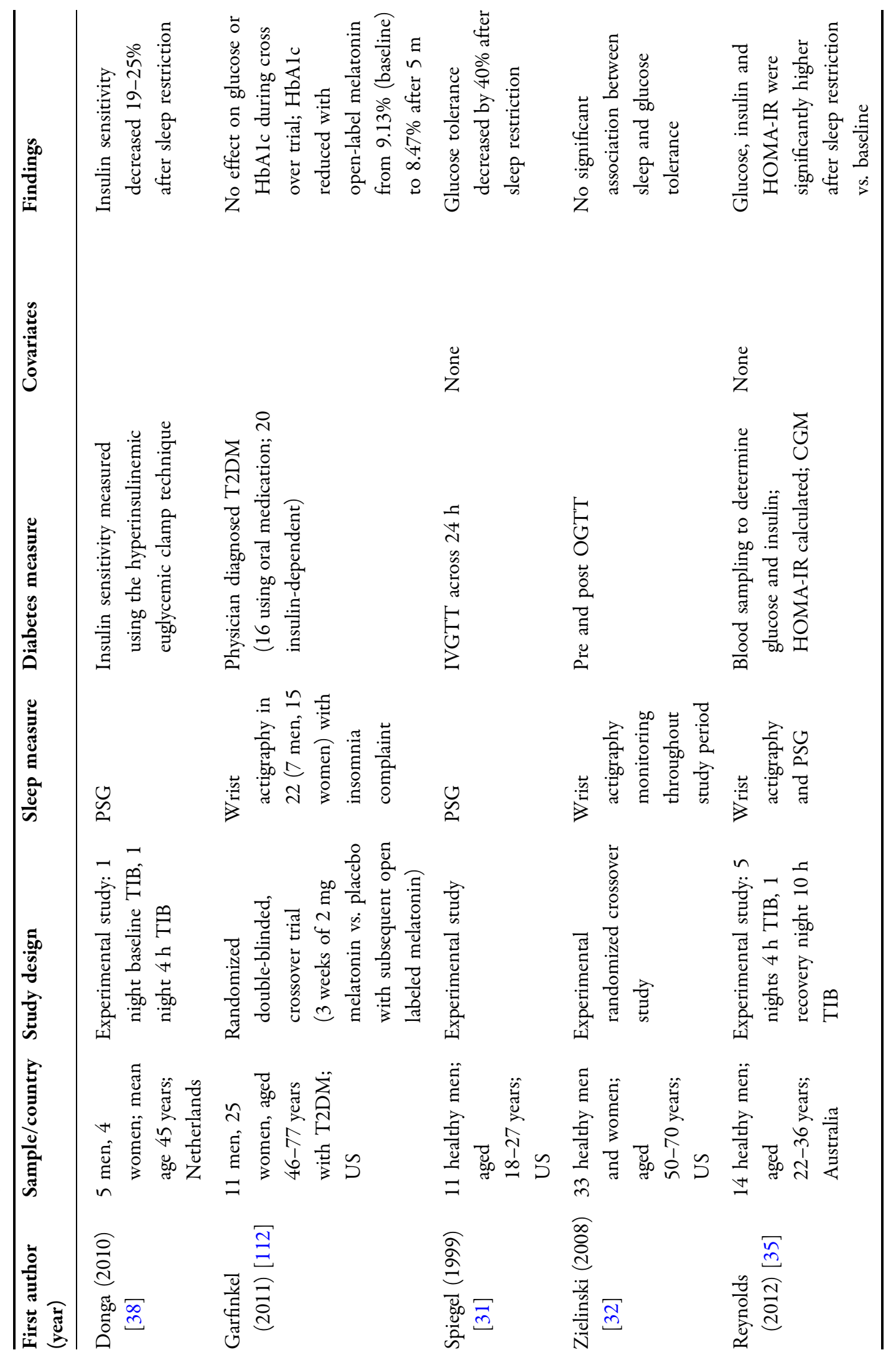




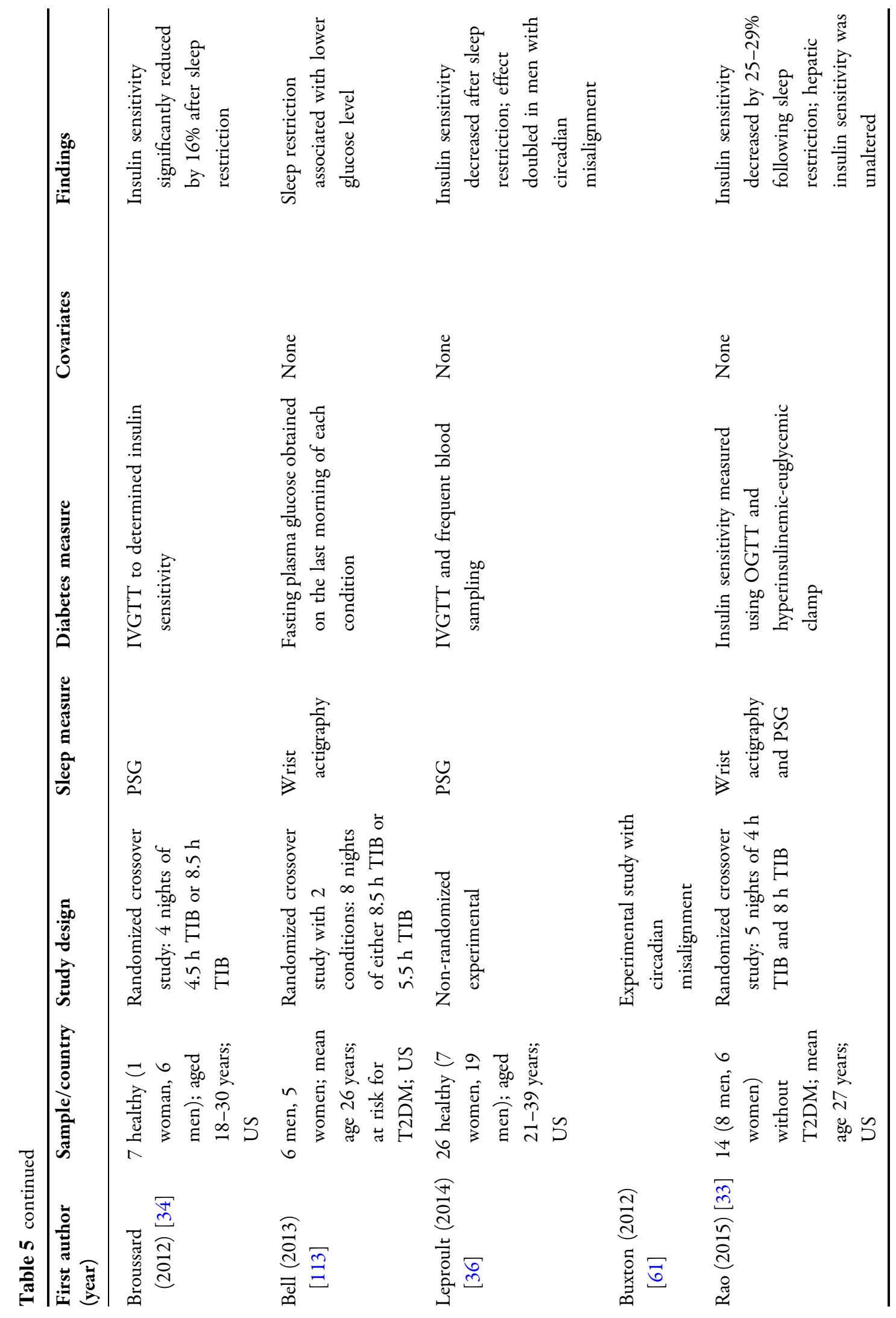




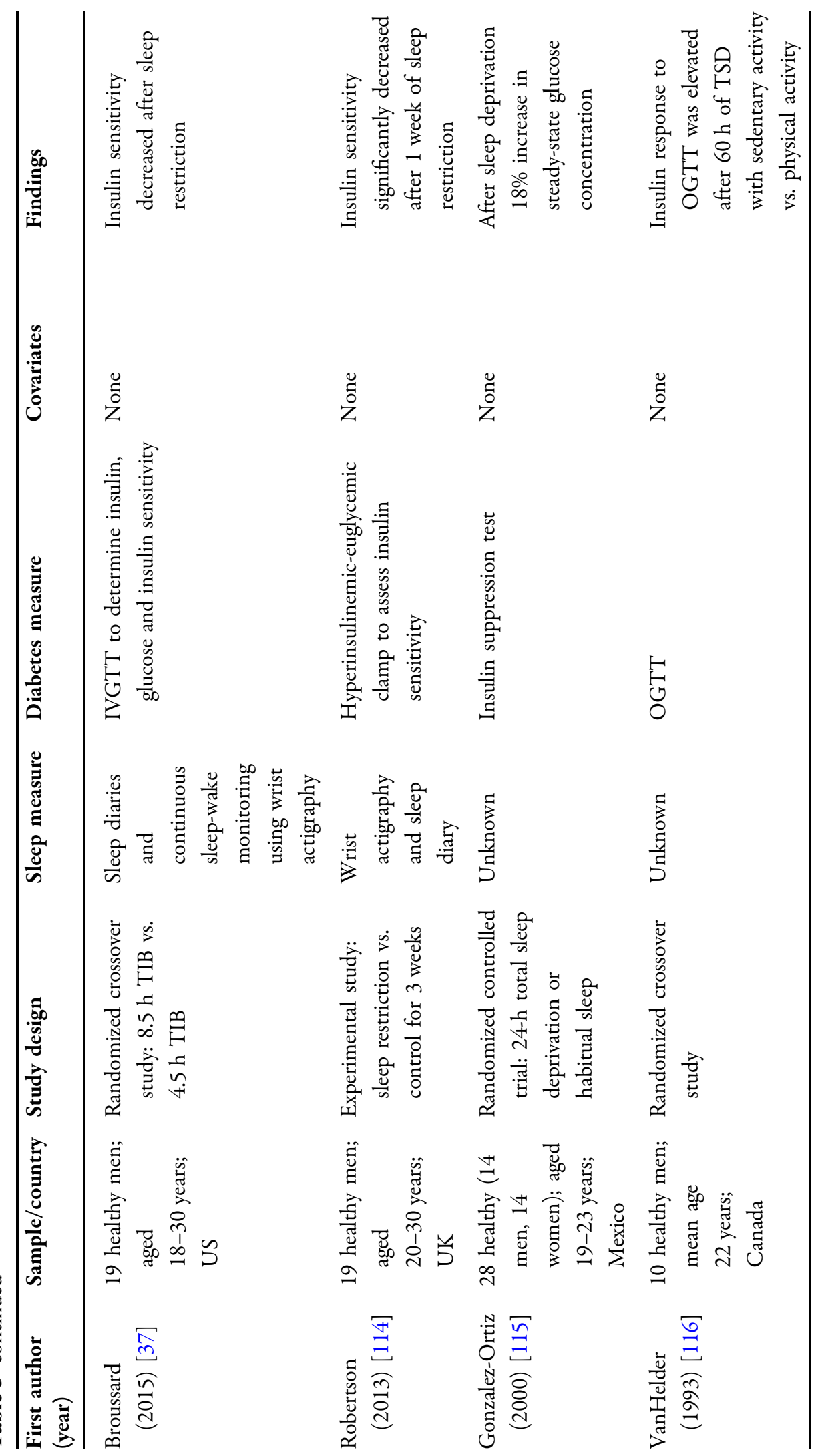




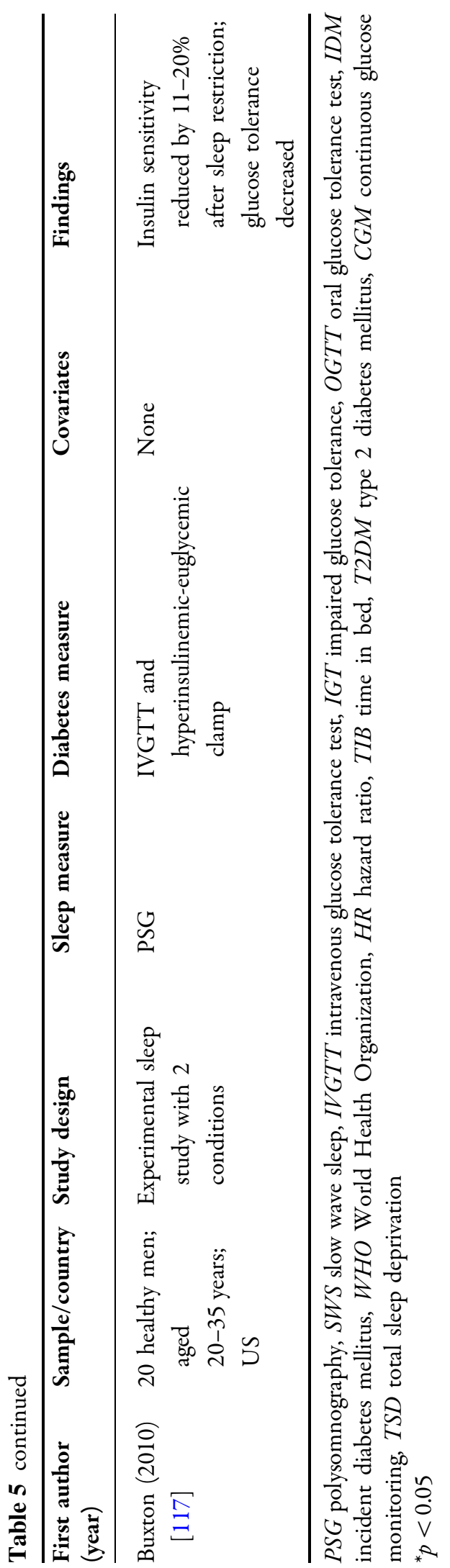

Spiegel and colleagues [31]. No significant associations were discovered between sleep condition and insulin resistance. There are various explanations, possibly from differences between study protocols, that may account for these observed discrepancies. First, volunteers were permitted to increase caffeine intake during sleep restriction to promote wakefulness [32]. Second, sleep restriction strategies were discussed between the volunteer and researcher to ensure facilitation, which may have resulted in different approaches to different individuals according to circadian preference that were not obtained and therefore not adjusted for within the analyses [32]. Third, a number of individuals completed the OGTT 2-5 days after the end of the 8-week manipulation [32]. These volunteers were instructed to continue with the allocated TIB until the OGTT had been performed, but reporting on continued compliance was not documented and may have biased the findings. Furthermore, extended time periods beyond the experimental period may have resulted in differences, although comparisons were not made in the analyses for these subjects. Fourth, a reduction of $90 \mathrm{~min}$ in Zielinski's protocol [32] may be inadequate to observe a significant effect and is less extreme than Spiegel's 4-h TIB [31]. Finally, the study recruited older individuals with a prolonged amount of TIB $(9 \mathrm{~h})$ versus total sleep time (7.4 h) [32], as compared to young healthy men in Spiegel's study [31].

Recent detailed study of hepatic and peripheral insulin sensitivity, as well as substrate utilization, in response to sleep restriction has been investigated. Rao and colleagues have revealed that an acute bout of extreme sleep reduction can result in profound metabolic alterations [33]. Participants received 2 nights of laboratory acclimatization followed 
by two conditions in a randomized crossover approach undertaking 5 nights of sleep restriction ( $4 \mathrm{~h}$ TIB) and normal sleep ( $8 \mathrm{~h}$ TIB), monitored using ambulatory PSG. At the end of the 5-night sleep restriction period, peripheral and whole-body insulin sensitivity significantly decreased by $29 \%$ and $25 \%$, respectively. No changes were noted for hepatic insulin sensitivity using the gold-standard hyperinsulinemic-euglycemic clamp technique [33]. An OGTT was also administered and HOMA-IR calculated, which revealed a $20 \%$ increase in insulin secretion following sleep restriction [33]. The authors of other experimental studies have reported comparable findings [34-37]. Similar effect sizes have also been reported following just 1 night of sleep reduction (4-h TIB) [38], suggesting that acute, extreme sleep restriction may result in metabolic disruption and a predisposition to diabetes.

The advantages of experimental sleep studies are that they usually benefit from the gold-standard measure to monitor sleep and incorporate accurate diabetes outcomes under very tightly controlled conditions, thereby removing the effect of known confounders. However, laboratory attendance may interfere with the natural sleep patterns and/or architecture, thereby introducing potential biases including environmental change (room temperature, altered noise/lighting, different bed comfort and more) and equipment to monitor sleep and diabetes (PSG wiring, catheters for repeated blood sampling). While laboratory studies are usually conducted under strictly controlled conditions, this may alter naturally occurring behaviors during attendance and objectively determined retrospective aspects of participant lifestyles and/or previous related events may not be considered but are crucial and may influence the findings. Furthermore, extreme sleep restriction is usually enforced but is not representative of voluntary small amounts of sleep loss experienced in societal behaviors. Finally, the majority of experimental work has been performed in healthy, young and male participants; therefore, the findings may not extend to other groups. That said, results of experimental sleep studies coupled with findings from large observational studies suggest a definitive association between sleep loss and diabetes. PSG monitoring confirms adherence to sleep manipulation by determining the total sleep time, but the authors do not usually report on other sleep features, despite the availability of output. Although the majority have focused on sleep duration and/or sleep loss, other sleep characteristics have also emerged to be important.

\section{Sleep Quality in Relation to Insulin Resistance and Diabetes}

Severely short sleep, defined by some as $\leq 5 \mathrm{~h}$ per night, as well as insomnia has been previously linked to a significantly increased risk of T2DM [18] and increased insulin resistance [39] through validated sleep measures. Lack of self-reported sleep [40] as well as sleep debt [41] has also been linked to the condition. These sleep outcomes (insomnia, lack of sleep and sleep debt) may be due to poor-quality sleep, which has resulted in researchers investigating this in relation to diabetes.

The most commonly used validated sleep quality questionnaire is the Pittsburgh Sleep Quality Index (PSQI). It is 24-item survey with 7 subcategories (sleep duration, disturbance, medication, latency, quality and efficiency as well as daytime dysfunction) that can be totaled 
to provide an overall score [42]. A combined score of more than five is suggestive of poor sleep quality. Some have investigated the prevalence of sleep quality, as determined by the PSQI, among T2DM. Fiorentini and colleagues reported a higher prevalence of T2DM in those with poor-quality sleep (19.4\%) compared to those with good quality sleep (8.8\%) [43]. Similarly, another study reported $33.3 \%$ of 50 participants with an HbA1c level of $>7 \%$ had poor quality sleep [44]. Another reported 55\% of 300 T2DM patients had poor-quality sleep [45]. Further, a sample of 551 patients in China revealed that the prevalence of poor sleep quality was significantly greater in those with poorer glycemic control and that average insulin resistance was higher among those with poorer sleep quality [16]. A larger effect size was observed by Tsai et al. using HbA1c levels to compare sleep quality using the PSQI [46]. After adjustment for age, gender and BMI in a sample of $46 \mathrm{~T} 2 \mathrm{DM}$ patients, poor sleep quality was associated with poorer glycemic control OR 6.94, $p=0.002$ [46]. Small studies with subjective sleep quality measures may be the result of chronic complications or 'side effects' of T2DM. In an attempt to disentangle the chronic impact of diabetes on sleep, Rajendran and colleagues administered the PSQI to investigate the relationship between sleep quality and duration of T2DM in 120 patients. Diabetes duration was negatively associated with the global PSQI score $(\beta=-020$, $p=0.02$ ), independent of age, gender, BMI, HbA1c and medication [47]. This association is consistent with another larger study $(n=614$ older patients with T2DM) [48].

Aside from the existing data from diabetic populations, the relationship between sleep quality and insulin resistance has also been investigated in healthy individuals as well as those at risk of developing T2DM. Hung et al. examined 1805 participants, of whom 1217 had normal glucose tolerance (healthy) and 118 had impaired fasting glucose (IFG), impaired glucose tolerance (IGT) was present in 287 , a total of 80 had both IFG and IGT, and 103 had newly diagnosed T2DM [49]. Comparisons among these groups were made according to sleep quality using the PSQI. The IGT group, compared to the healthy group, was positively associated with global PSQI when assessed through linear regression after adjustment for a range of confounders including self-reported physical activity, BMI, demographics, lipids, blood pressure and lifestyle factors, $\beta=0.63$ (95\% CI 0.33-0.94). A similar observation was reported for individuals with IFG and IGT, compared to the healthy group, where $\beta=0.61 \quad$ (95\% CI 0.06-1.15). The greatest effect size for poorer sleep quality was present in those with T2DM versus healthy individuals, $\beta=0.86$ (95\% CI 0.37-1.35). PSQI was then dichotomized into poor quality $(>5)$ and good quality $(\leq 5)$ was used as the referent category. Comparing all groups to the healthy group, only those with an existing diagnosis of T2DM showed a significant effect of poor sleep quality, OR 2.27 (95\% CI 1.39-3.70), after adjustment [49]. Although cross sectional, this study partly controls for the potential chronic and psychological impact of diabetes on sleep given that those with diabetes were unaware of their diagnosis.

A broad range of characteristics can be used to measure sleep quality. Sleep efficiency, sleep fragmentation, number of wake episodes, wake after sleep onset and length of awakenings are all indicators. Sleep efficiency (the percentage of time spent in bed sleeping) has been used to explore the relationship with diabetes. The Coronary Artery Risk Development in young Adults (CARDIA) study recruited young 
individuals (aged $18-30$ years) with $(n=40)$ and without $(n=115)$ T2DM [39]. A fasting blood sample was drawn, and insulin resistance was measured using HOMA-IR. A wrist actigraphy device was worn for 3 consecutive days/nights and repeated around 2 years later to estimate sleep fragmentation, an indicator of sleep quality. Insulin resistance was positively and significantly associated with sleep fragmentation in those with T2DM only before and after adjustment, where $\beta=0.36, p<0.001$ and $\beta=0.35, p<0.001$, respectively. Insomnia, but not sleep duration, was positively associated with insulin resistance in those with T2DM but not those without [39]. The relationship between sleep fragmentation and insulin sensitivity has been confirmed by others in case-control studies [50, 51] as well as experimental manipulation [52]. Two nights of induced sleep fragmentation in controlled laboratory conditions has been shown to significantly decrease insulin sensitivity by $25.2 \%$ and glucose effectiveness by $20.9 \%$ [52]. Sleep fragmentation is a common feature of sleep-disordered breathing (SDB), specifically obstructive sleep apnea (OSA), a condition closely linked to T2DM (for brief discussion, see the section titled "The role of other sleep features upon insulin resistance and diabetes").

The majority of studies highlighting a relationship between indicators of sleep quality and diabetes are based on cross-sectional data $[10,16,39,41,43-49$, 53-57], and while just one found no association [56], causality remains the main issue. A small number of case-control studies have aided comparisons of differences in sleep quality between those with and without T2DM $[50,51,58]$. There are, however, limited convincing prospective data. Early work by Nilsson et al. used a non-validated questionnaire (two questions) to determine the difficulty of initiating sleep and use of hypnotics along with subjectively reported T2DM with a mean follow-up of 15 years, reporting a $52 \%$ and $78 \%$ increased risk of incident T2DM in those with one or both sleep features, respectively [59]. Similar findings have been reported by those with difficulty maintaining sleep [53]. Conversely, another group found no association between difficulties initiating sleep and objectively confirmed incident T2DM [26]. Thus, recruitment of initially healthy, young populations incorporating regular prospective follow-ups paired with objective sleep/activity monitoring in the natural environment is required to confirm or refute the relationship between sleep quality and diabetes.

\section{The Emerging Effect of Sleep-Wake Misalignment Upon Insulin Resistance and Diabetes}

While the majority of focus has been on sleep quantity and quality relative to diabetes, there is now emerging evidence suggesting that circadian rhythms, chronotype and sleep-wake timings play an important role in diabetes onset, development and management. It is well established that shift workers have a higher prevalence of metabolic disorders [60]. In particular, night-shift workers and rotating shift workers are among those worst affected as they not only experience circadian disruption but also sleep loss. These extreme patterns are in conflict with human evolution and challenge our internal circadian pacemakers, regulated by the hypothalamic suprachiasmatic nucleus. Recent evidence suggests that extreme sleep-wake timings are not necessary to cause metabolic alteration, but that even slightly shifted changes and/or circadian preferences can influence diabetes. 
Buxton and colleagues examined the effects of sleep restriction combined with gradual circadian misalignment (eventually simulating shift-work patterns) upon glucose metabolism. A total of 21 healthy participants were examined across a 39 consecutive day/night period in a strictly controlled laboratory setting [61]. A 32\% reduction in insulin response to a standardized meal was reported, resulting in inadequate glucose regulation. The resting metabolic rate was also reduced following experimental manipulation of sleep restriction and circadian desynchrony, although levels reverted back to baseline subsequent to 9 days/ nights of recovery sleep [61].

Leproult et al. examined a healthy population of young (21-39 years) individuals $(n=26)$. Wrist actigraphy was used to monitor standardized sleep-wake schedules 1 week prior to the circadian misalignment intervention [36]. Upon laboratory attendance, sleep was monitored using PSG and volunteers underwent 3 baseline days/nights, then 8 days/nights of $5 \mathrm{~h}$ TIB (centered around 03:00: circadian alignment). Half of the sample experienced an 8.5-h delay in bedtimes (09:00-14:00) for 4 of the 8 nights (circadian misalignment), followed by 3 nights of sleep recovery. All participants encountered the same amount of sleep opportunity ( $24 \mathrm{~h}$ over 8 days/nights), ensuring the effect of circadian misalignment per se was examined. An IVGTT was performed following an overnight fast on the 2nd baseline day as well as on the 2nd to last day of the intervention, and multiple blood samples were obtained. Insulin sensitivity was decreased in $96 \%$ of the sample, which was unaccompanied by an increase in responsiveness of $\beta$-cell function. The decrease in insulin sensitivity was almost double in the misaligned group $(-58 \%)$ versus the controls $(-32 \%), p=0.011$. Similarly, a greater effect was observed in the experimental group where C-reactive protein (an indicator of systemic inflammation) increased by $146 \%$ from baseline compared to the control group (+64\%).

Taken together, findings from enforced circadian misalignment concurrent with sleep loss provide clues about metabolic alterations that arise from sleep changes, which challenge human physiology. It is well known that physical activity is crucial for preventing diabetes onset and progression. If sleep loss paired with circadian misalignment results in decreases in basal metabolic rate then a greater amount of energy expenditure will be required for energy homeostasis. Additional energy expenditure is less likely when in a sleep-deprived state. Furthermore, sleep-wake timings that are inconsistent with human evolution may result in decreased insulin sensitivity as well as increased systemic inflammation, which is associated with cardiovascular disease. Forced desynchronization is extreme and simulates shift work but is not necessarily representative of societal behaviors or day workers. Other groups have investigated less radical approaches of circadian misalignment by examining circadian preferences (morningness-eveningness). A number of studies have investigated circadian preference in T2DM patients with cross-sectional data [12, 62-65].

One group highlighted a significant association between $\mathrm{HbA} 1 \mathrm{c}$ and self-reported circadian preference, where $\beta=-1.54, p=0.03$ after adjustment for age and high-density lipoprotein cholesterol in 101 Japanese men [65]. Lower scores on the morningness-eveningness questionnaire (MEQ) indicate evening types, suggesting that 'night owls' had poorer glycemic control [65]. Another study included 725 Japanese mixed-gender 
T2DM patients and found a dose-dependent effect of circadian preference on HbA1c levels. HbA1c was significantly higher in those with evening preference (7.3\%) compared to morning types $(6.7 \%)$, and linear regression revealed $\beta=-2.94, p<0.01$ after adjustment for a range of established potential confounders including physical activity, dietary intake and demographics [64]. One further study that recruited a random sample of 6,258 Finnish adults, aged $25-74$ years, revealed that evening types (determined from MEQ) predicted increased odds of T2DM (OR 2.6, $p<0.0001$ ), after adjustment [12]. Two more studies by the same group, and possibly data analysis from the same sample, demonstrated significant evening chronotype associations with diabetes $[62,63]$. A total of 194 T2DM patients were assessed using the midpoint of sleep, calculated from self-reported estimated sleep-wake times in order to determine circadian preference (chronotype). Glycemic control (HbA1c) was obtained from medical records, and the relationship was assessed with circadian preference. The midpoint of sleep on free days was positively correlated $(r=0.34, p<0.001)$ with glycemic control. In hierarchial regression analysis, the association remained, where $\beta=0.03, \quad p=0.001$ after adjustment [63]. Similar findings were reported in the other study where it was proposed that chronotype mediated the association between skipping breakfast and glycemic control [62].

Aside from experimental work, insulin sensitivity in relation to circadian misalignment/preference has not been extensively examined, although it is a growing area of study. The existing evidence indicates that extreme misalignment of sleep-wake timings in healthy individuals may promote pre-diabetes. Furthermore, evening types with T2DM may have poorer glycemic control, although causality remains undetermined, and further investigation is required for confirmation.

\section{The Role of Other Sleep Features Upon Insulin Resistance and Diabetes}

When sleep occurs, highly complex physiological processes ensue including hormone release, information processing, cellular restoration and more. Previously, sleep was believed to be a phenomenon for and by the brain. Undoubtedly, the brain regulates sleep, but evidence now indicates clear peripheral effects on metabolic outcomes. This is representative of gradual sleep alterations that have occurred within contemporary society, concomitant with the rising prevalence of metabolic diseases. Much of the research focus has been on sleep quantity; however, there are many other significant sleep characteristics that have been liked to T2DM.

Frequent and longer daytime sleep (napping/ siesta) has been linked to a higher risk of T2DM prevalence/incidence [66-68]. It has been suggested that napping may be a consequence of poor sleep quality and/or insufficient sleep duration. In the studied populations, however, napping is habitual and believed to have beneficial health effects. Napping after lunch reflects the human circadian rhythm (post-lunch dip) although, according to the two-process model of sleep [1], there is insufficient prior wakefulness to warrant sleep initiation. Some have therefore considered daytime napping and combined this with nocturnal sleep duration (calculating total sleep time) to investigate the relationship between total sleep and metabolic outcomes $[69,70]$. Experimental sleep studies, however, usually prohibit napping in study protocols and thus may not capture crucial information. 
There are many documented metabolic consequences of daytime sleep/circadian misalignment $[36,61,71]$, with some linking this to diabetes complications [72]. Sleep debt is usually accumulated across the week and repaid during weekends. Recent experimental data in 19 healthy males with at least a 6-month history of voluntary sleep curtailment were recruited. When 'catch-up' sleep was not permitted and short sleep persisted across a 7-day period for 3 weeks, insulin sensitivity decreased and HOMA-IR increased compared to those with compensatory weekend catch-up sleep [73].

Snoring may also be a risk factor for T2DM [74], possibly as a consequence of OSA. Undoubtedly, OSA is strongly linked to diabetes, although which develops first is to be determined, given that they are both noncommunicable. Obstructive sleep apnea (OSA), as well as concomitant intermittent nocturnal hypoxemia, is common in patients with T2DM [75, 76]. Several studies have reported an association between OSA, glycemic control and diabetes microvascular complications [77-81]. OSA appears to be more common in ethnicities where T2DM is more common [82]. Many studies have not been able to address the confounding effect of OSA in the relationship between sleep and metabolism. Interestingly, continuous positive airway pressure (CPAP), used to treat OSA, has also been shown to improve glycemic control prospectively [75]. Thus, improving sleep can in turn improve metabolic profiles. While SDB certainly disrupts metabolic regulation and extensive data have recently emerged in relation to insulin resistance and other features of diabetes, this is beyond the scope of the current review. Clearly, the extent of other sleep aspects on metabolic health is slowly emerging, although a better understanding of all characteristics is still required, along with mechanistic explanations.

Significant changes in sleep architecture have also been noted in a retrospective case-control study [58]. T2DM patients had $4.5 \%$ less slow wave sleep (SWS) and 10.3\% more rapid eye movement (REM) sleep compared to those without the condition. Differences in sleep architecture between those with and without diabetes have also been investigated in the Sleep Heart Healthy Study but detected different results [83]. Differences in SWS between diabetic and non-diabetic volunteers diminished after adjustment, and REM sleep accounted for a significantly reduced proportion of total sleep time in those with diabetes [83]. Tasali and colleagues attempted to disentangle this relationship by suppressing SWS for 3 consecutive nights using an acoustic stimulus in nine healthy, young volunteers [84]. SWS suppression was achieved and resulted in significant reductions in insulin sensitivity $(\sim 25 \%)$ and glucose tolerance $(\sim 23 \%)$ compared to baseline. SWS is considered to have a restorative effect; thus, a reduction in this deep phase of sleep may induce metabolic alterations that can promote diabetes as well as other related comorbidities. SWS declines with age and diabetes risk increases with age; thus, age may mediate this relationship, and future studies need to investigate the strength of such associations in older individuals as well as those with T2DM.

\section{Mechanisms}

There is now clear evidence linking sleep to the onset and development of pre-diabetes and T2DM. Understanding the mechanisms involved is of great importance. It has been suggested that insufficient sleep and/or poor quality sleep can result in oxidative stress as 
well as overactivation of the sympathetic nervous system, and some have used heart rate variability to support this notion [31]. Others have shown that changes in the appetite-regulating hormones leptin (related to satiety) and ghrelin (related to hunger) have been observed in response to short/insufficient sleep duration [85, 86]. Disruption of the regulation of these hormones from sleep loss has also been linked to an increased appetite for carbohydrate-dense foods $[85,86]$ and intake of calories from sweet foods (snacks) [87]. Poor dietary habits are well known to promote T2DM; thus, the effects of sleep loss may contribute to these unhealthy behaviors through metabolic disruption. Sleep deprivation is associated with activation of the hypothalamic orexin (hypocretin) neuropeptide system [88-90]. Orexin (hypocretin) neurons are located in the lateral hypothalamus and project throughout the central nervous system and particularly to areas important in wakefulness. Orexin activation is associated with increased sympathetic nervous system activation, increased cortisol and suppressed growth hormone secretion, which can all predispose to hyperglycemia. Orexin receptor antagonists are currently under investigation for use in insomnia, and it would be of interest to study their impact on metabolism. The importance of the central nervous system in the regulation of glucose metabolism has been further highlighted by the use of bromocriptine in the treatment of T2DM. Bromocriptine is a dopamine D2 receptor agonist, primarily used in the treatment of prolactinoma. Bromocriptine has received United States Food and Drug administration approval for the treatment of T2DM. Its use is supported by more recent randomized controlled trials (RCTs) in those with diabetes [91, 92]. The precise site of action of bromocriptine in regulating metabolism is unknown, but dopamine acts at several levels including the hypothalamus and pineal gland [93], which are key areas for the regulation of circadian rhythms, sleep and metabolism. The timing of bromocriptine administration in glycemic regulation appears to be important with greater effects in the morning when endogenous dopaminergic drive is at its peak. A side effect of bromocriptine for diabetes treatment is sleepiness. Dopamine receptor antagonism by anti-psychotic drugs has been observed to be associated with obesity and subsequent insulin resistance and diabetes.

\section{Key Limitations}

Some observational data highlight a U-shaped relationship between sleep and diabetes prevalence, and while much of the focus has been on short sleepers, long sleep may also be problematic and has shown a greater effect size compared to short sleep in a recent meta-analysis of prospective studies [94]. Previously, conclusions suggested that long sleepers possibly have underlying health issue(s) that result in sleep lengthening. Of course, this may not always be true; thus, there is a need to identify general cut points for when sleep may become harmful, given that sleep extension studies are underway in an attempt to address metabolic dysregulation. Definitions of short/long sleep are also inconsistent, meaning comparisons can be difficult and conclusions misconstrued. There is a need for reliable cut points that can be applied across all studies to ensure uniformity.

Most of the sleep-diabetes evidence is consistent although not completely homogeneous. Of particular concern is the lack of prospective evidence for sleep quality 
and diabetes onset/progression. Other prospective discrepancies exist from a powerful 32-year follow-up study [27] finding no association between sleep and incident diabetes. There are some potential explanations for this including (1) the possibility of OSA as a hidden confounder, an unrecognized sleep disorder at the time the study commenced; (2) lack of an objective sleep measure; (3) gender differences, given that the study was only conducted in women; (4) dietary habits were not considered.

Sleep perceptions are subject to several biases; thus, objective measures in natural environments are preferable. Participants should be regularly monitored with validated wrist actigraphy to ensure sleep-wake information is captured. However, that said, sleep is not static, making it incredibly difficult to capture an accurate picture and study its effects. Furthermore, there is no gold-standard measure of dietary intake, an important confounder of the sleep-diabetes relationship. Capturing full and accurate information about food selection, micro-/macronutrients, portion sizes, methods of cooking, unknown ingredients in takeaway food and more is near impossible to reliably measure in a natural setting. Food diaries are a reasonable compromise but require specific programs to analyze the data, are time consuming to administer, complete and analyze, may not be completed, are subjective and can therefore introduce bias. An improved alternative that is accurate is urgently needed, given that energy intake/selection can dramatically alter metabolic regulation and hormone release. The majority of the work, whether observational, case-control, prospective or experimental, has not ascertained or adjusted for the combination of the three factors known to promote diabetes (energy intake, energy expenditure, familial history). This is a major limitation in the existing literature, and consistency across studies of all potential confounding factors is urgently needed.

Studies performed in diabetes patients usually adjust for diabetes duration and medications. Details of medications are usually omitted, but specifications (name, frequency, dosage and timings) are likely to be important, particularly for medications shown to alter sleep. One further consideration is the possibility of psychological conditions, which have sometimes been adjusted for in sleep-diabetes studies. However, medication related to these conditions, which can influence sleep-wake behaviors, as well as the condition itself should be considered. Commonly used antidepressants, for example, have significant effects on sleep architecture. The impact of medications on the sleep-diabetes relationship can also be complex. For example, pregabalin enhances sleep by itself and also reduced sleep disturbance secondary to a reduction in neuropathic pain in diabetes [95]. In some patients, however, significant weight gain may occur with pregabalin and other similar drugs, which increases insulin resistance.

Finally, the majority of the experimental work has been conducted in young, healthy individuals, with more data available in men, given the complexities of the female menstrual cycle that may interfere with metabolic hormone measurements. While findings from these types of studies have highlighted important information and provided a foundation for our understanding, detailed work is required in those with T2DM as well as older individuals so that we can establish potential interventions for improving glucose control in those with long-standing disease or reverse the condition in those with new diagnoses. 


\section{Future Directions}

Interventions that target those with T2DM, as well as prevention of its onset, should incorporate sleep education in addition to energy balance advice. Emerging evidence from a small study of healthy volunteers randomized to sleep extension for 40 days/ nights or control has provided preliminary but promising results for improving insulin sensitivity with as little as one additional hour of time in bed [96]. Future studies should repeat this sleep extension/improvement of sleep quality in those with T2DM for further comprehensive examination and understanding. After all, making healthy food selection, controlling portion sizes and achieving the recommended minimum $30 \mathrm{~min}$ of daily activity require much dedication and willpower. Conversely, compliance with staying in bed for an extra hour may be easier and costs the individual little, but may greatly benefit his or her metabolic profile and could prevent the onset and/or progression of diabetes.

The majority of sleep studies in relation to diabetes mellitus have focused on T2DM. There is limited information on those with type 1 diabetes, although recent preliminary evidence has emerged suggesting that multiple sleep parameters as well as sleep staging play a role in glycemic control in this population $[97,98]$. Sleep may play a key role in other types of diabetes mellitus although little is known about this, warranting further investigation.

\section{CONCLUSION}

The current evidence suggests that sleep is instrumental to metabolic regulation and disease management. Disruption of sleep homeostasis, which is comprised of multiple components (quantity, quality, timing and architecture), can result in adverse metabolic consequences. The effect of disruption of one component has been established, but disruption of multiple sleep features may worsen diabetes control, although this requires further investigation. Sleep imbalance may promote diabetes onset or hinder glucose control and insulin sensitivity in those with pre-existing diabetes. Cross-sectional studies as well as prospective cohort findings demonstrate reasonably consistent findings and implicate a role for sleep in the management of diabetes. Furthermore, acute sleep disruption under controlled laboratory conditions has shown significant and negative effects upon glucose control in healthy adults. Exposure to persistent sleep imbalance is likely to be detrimental to metabolic health/disease status. While the evidence is convincing, a number of limitations are present, including the possibility of uncontrolled major confounders, which restrict robust conclusions. Further investigation in 'at risk' populations as well as those with T2DM is needed incorporating objective and prospective sleep measures. It may be possible to prevent incident diabetes and smooth the current epidemic by improving diabetes control through sleep optimization in combination with other lifestyle advice, particularly in newly diagnosed cases. Promoting the importance of sleep for improving diabetes control/management is unlikely to result in any harmful consequences. Awareness of an additional, and easily modifiable, lifestyle behavior among healthcare professionals is therefore recommended. 


\section{ACKNOWLEDGMENTS}

Both authors are funded by the Biomedical Research Program (BMRP) at Weill Cornell Medical College in Qatar, supported by the Qatar Foundation. No funding was received for the article processing charges. Teresa Arora and Shahrad Taheri designed the review. Teresa Arora drafted the initial article, and Shahrad Taheri critically revised the intellectual content. All named authors meet the International Committee of Medical Journal Editors (ICMJE) criteria for authorship for this manuscript, take responsibility for the integrity of the work as a whole, and have given final approval for the version to be published.

Disclosures. Teresa Arora and Shahrad Taheri declare that they have no conflict of interest.

Compliance with ethical guidelines. This article is based on previously conducted studies and does not involve any new studies of human or animal subjects performed by either of the authors.

Open Access. This article is distributed under the terms of the Creative Commons Attribution-NonCommercial 4.0 International License (http://creativecommons.org/licenses/ by-nc/4.0/), which permits any noncommercial use, distribution, and reproduction in any medium, provided you give appropriate credit to the original author(s) and the source, provide a link to the Creative Commons license, and indicate if changes were made.

\section{REFERENCES}

1. Borbely AA. A two process model of sleep regulation. Hum Neurobiol. 1982;1(3):195-204.
2. Taheri S, Mignot E. Sleep well and stay slim: dream or reality? Ann Intern Med. 2010;153(7):475-6.

3. Gottlieb DJ, Punjabi NM, Newman AB, Resnick HE, Redline S, Baldwin CM, et al. Association of sleep time with diabetes mellitus and impaired glucose tolerance. Arch Intern Med. 2005;165(8):863-7.

4. Chaput JP, Despres JP, Bouchard C, Tremblay A. Association of sleep duration with type 2 diabetes and impaired glucose tolerance. Diabetologia. 2007;50(11):2298-304.

5. Tuomilehto H, Peltonen M, Partinen M, Seppa J, Saaristo T, Korpi-Hyovalti E, et al. Sleep duration is associated with an increased risk for the prevalence of type 2 diabetes in middle-aged women-the FIN-D2D survey. Sleep Med. 2008;9(3):221-7.

6. Kim J, Kim HM, Kim KM, Kim DJ. The association of sleep duration and type 2 diabetes in Korean male adults with abdominal obesity: the Korean National Health and Nutrition Examination Survey 2005. Diabetes Res Clin Pract. 2009;86(2):e34-6.

7. Chao CY, Wu JS, Yang YC, Shih CC, Wang RH, Lu $\mathrm{FH}$, et al. Sleep duration is a potential risk factor for newly diagnosed type 2 diabetes mellitus. Metab Clin Exp. 2011;60(6):799-804.

8. Liu R, Zee PC, Chervin RD, Arguelles LM, Birne J, Zhang S, et al. Short sleep duration is associated with insulin resistance independent of adiposity in Chinese adult twins. Sleep Med. 2011;12(9):914-9.

9. Kachi Y, Ohwaki K, Yano E. Association of sleep duration with untreated diabetes in Japanese men. Sleep Med. 2012;13(3):307-9.

10. Lou P, Chen P, Zhang L, Zhang P, Yu J, Zhang N, et al. Relation of sleep quality and sleep duration to type 2 diabetes: a population-based cross-sectional survey. BMJ Open. 2012;2(4). doi:10.1136/ bmjopen-2012-000956

11. Ohkuma T, Fujii H, Iwase M, Kikuchi Y, Ogata S, Idewaki $Y$, et al. Impact of sleep duration on obesity and the glycemic level in patients with type 2 diabetes: the Fukuoka Diabetes Registry. Diabetes Care. 2013;36(3):611-7.

12. Merikanto I, Lahti T, Puolijoki $H$, Vanhala $M$, Peltonen M, Laatikainen T, et al. Associations of chronotype and sleep with cardiovascular diseases and type 2 diabetes. Chronobiol Int. 2013;30(4):470-7.

13. Najafian J, Mohamadifard N, Siadat ZD, Sadri G, Rahmati MR. Association between sleep duration 
and diabetes mellitus: Isfahan Healthy Heart Program. Niger J Clin Pract. 2013;16(1):59-62.

14. Kim BK, Kim BS, An SY, Lee MS, Choi YJ, Han SJ, et al. Sleep duration and glycemic control in patients with diabetes mellitus: Korea National Health and Nutrition Examination Survey 2007-2010. J Korean Med Sci. 2013;28(9):1334-9.

15. Wong PM, Manuck SB, DiNardo MM, Korytkowski M, Muldoon MF. Shorter sleep duration is associated with decreased insulin sensitivity in healthy white men. Sleep. 2015;38(2):223-31.

16. Tang Y, Meng L, Li D, Yang M, Zhu Y, Li C, et al. Interaction of sleep quality and sleep duration on glycemic control in patients with type 2 diabetes mellitus. Chin Med J. 2014;127(20):3543-7.

17. Zuo H, Shi Z, Yuan B, Dai Y, Hu G, Wu G, et al. Interaction between physical activity and sleep duration in relation to insulin resistance among non-diabetic Chinese adults. BMC Public Health. 2012;12:247.

18. Vgontzas AN, Liao D, Pejovic S, Calhoun S, Karataraki M, Bixler EO. Insomnia with objective short sleep duration is associated with type 2 diabetes: a population-based study. Diabetes Care. 2009;32(11):1980-5.

19. Darukhanavala A, Booth JN 3rd, Bromley L, Whitmore H, Imperial J, Penev PD. Changes in insulin secretion and action in adults with familial risk for type 2 diabetes who curtail their sleep. Diabetes Care. 2011;34(10):2259-64.

20. Heianza Y, Kato K, Fujihara K, Tanaka S, Kodama $\mathrm{S}$, Hanyu $\mathrm{O}$, et al. Role of sleep duration as a risk factor for Type 2 diabetes among adults of different ages in Japan: the Niigata Wellness Study. Diabet Med J Br Diabet Assoc. 2014;31(11):1363-7.

21. Gangwisch JE, Heymsfield SB, Boden-Albala B, Buijs RM, Kreier F, Pickering TG, et al. Sleep duration as a risk factor for diabetes incidence in a large US sample. Sleep. 2007;30(12):1667-73.

22. Boyko EJ, Seelig AD, Jacobson IG, Hooper TI, Smith B, Smith TC, et al. Sleep characteristics, mental health, and diabetes risk: a prospective study of US military service members in the Millennium Cohort Study. Diabetes Care. 2013;36(10):3154-61.

23. Holliday EG, Magee CA, Kritharides L, Banks E, Attia J. Short sleep duration is associated with risk of future diabetes but not cardiovascular disease: a prospective study and meta-analysis. PLoS One. 2013;8(11):e82305.
24. Ayas NT, White DP, Al-Delaimy WK, Manson JE, Stampfer MJ, Speizer FE, et al. A prospective study of self-reported sleep duration and incident diabetes in women. Diabetes Care. 2003;26(2):380-4.

25. Yaggi HK, Araujo AB, McKinlay JB. Sleep duration as a risk factor for the development of type 2 diabetes. Diabetes Care. 2006;29(3):657-61.

26. Hayashino Y, Fukuhara S, Suzukamo Y, Okamura T, Tanaka T, Ueshima $\mathrm{H}$, et al. Relation between sleep quality and quantity, quality of life, and risk of developing diabetes in healthy workers in Japan: the High-risk and Population Strategy for Occupational Health Promotion (HIPOP-OHP) Study. BMC Public Health. 2007;7:129.

27. Bjorkelund C, Bondyr-Carlsson D, Lapidus L, Lissner L, Mansson J, Skoog I, et al. Sleep disturbances in midlife unrelated to 32-year diabetes incidence: the prospective population study of women in Gothenburg. Diabetes Care. 2005;28(11):2739-44.

28. Gutierrez-Repiso C, Soriguer F, Rubio-Martin E, Esteva de Antonio I, Ruiz de Adana MS, Almaraz $\mathrm{MC}$, et al. Night-time sleep duration and the incidence of obesity and type 2 diabetes. Findings from the prospective Pizarra study. Sleep Med. 2014;15(11):1398-404.

29. Beihl DA, Liese AD, Haffner SM. Sleep duration as a risk factor for incident type 2 diabetes in a multiethnic cohort. Ann Epidemiol. 2009;19(5):351-7.

30. Chaput JP, Despres JP, Bouchard C, Astrup A, Tremblay A. Sleep duration as a risk factor for the development of type 2 diabetes or impaired glucose tolerance: analyses of the Quebec Family Study. Sleep Med. 2009;10(8):919-24.

31. Spiegel K, Leproult R, Van Cauter E. Impact of sleep debt on metabolic and endocrine function. Lancet. 1999;354(9188):1435-9.

32. Zielinski MR, Kline CE, Kripke DF, Bogan RK, Youngstedt SD. No effect of 8-week time in bed restriction on glucose tolerance in older long sleepers. J Sleep Res. 2008;17(4):412-9.

33. Rao MN, Neylan TC, Grunfeld C, Mulligan K, Schambelan M, Schwarz JM. Subchronic sleep restriction causes tissue-specific insulin resistance. J Clin Endocrinol Metab. 2015;100(4):1664-71.

34. Broussard JL, Ehrmann DA, Van Cauter E, Tasali E, Brady MJ. Impaired insulin signaling in human adipocytes after experimental sleep restriction: a 
randomized, crossover study. Ann Intern Med. 2012;157(8):549-57.

35. Reynolds AC, Dorrian J, Liu PY, Van Dongen HP, Wittert GA, Harmer LJ, et al. Impact of five nights of sleep restriction on glucose metabolism, leptin and testosterone in young adult men. PLoS One. 2012;7(7):e41218.

36. Leproult R, Holmback U, Van Cauter E. Circadian misalignment augments markers of insulin resistance and inflammation, independently of sleep loss. Diabetes. 2014;63(6):1860-9.

37. Broussard JL, Chapotot F, Abraham V, Day A, Delebecque F, Whitmore HR, et al. Sleep restriction increases free fatty acids in healthy men. Diabetologia. 2015;58(4):791-8.

38. Donga E, van Dijk M, van Dijk JG, Biermasz NR, Lammers GJ, van Kralingen KW, et al. A single night of partial sleep deprivation induces insulin resistance in multiple metabolic pathways in healthy subjects. J Clin Endocrinol Metab. 2010;95(6):2963-8.

39. Knutson KL, Van Cauter E, Zee P, Liu K, Lauderdale DS. Cross-sectional associations between measures of sleep and markers of glucose metabolism among subjects with and without diabetes: the Coronary Artery Risk Development in Young Adults (CARDIA) Sleep Study. Diabetes Care. 2011;34(5):1171-6.

40. Andersson S, Ekman I, Friberg F, Bog-Hansen E, Lindblad $U$. The association between self-reported lack of sleep, low vitality and impaired glucose tolerance: a Swedish cross-sectional study. BMC Public Health. 2013;13:700.

41. Knutson KL, Ryden AM, Mander BA, Van Cauter E. Role of sleep duration and quality in the risk and severity of type 2 diabetes mellitus. Arch Intern Med. 2006;166(16):1768-74.

42. Buysse DJ, Reynolds CF 3rd, Monk TH, Berman SR, Kupfer DJ. The Pittsburgh Sleep Quality Index: a new instrument for psychiatric practice and research. Psychiatry Res. 1989;28(2):193-213.

43. Fiorentini A, Valente R, Perciaccante A, Tubani L. Sleep's quality disorders in patients with hypertension and type 2 diabetes mellitus. Int J Cardiol. 2007;114(2):E50-2.

44. Cunha MC, Zanetti ML, Hass VJ. Sleep quality in type 2 diabetics. Rev Lat Am Enfermagem. 2008;16(5):850-5.

45. Luyster FS, Dunbar-Jacob J. Sleep quality and quality of life in adults with type 2 diabetes. Diabetes Educ. 2011;37(3):347-55.
46. Tsai YW, Kann NH, Tung TH, Chao YJ, Lin CJ, Chang KC, et al. Impact of subjective sleep quality on glycemic control in type 2 diabetes mellitus. Fam Pract. 2012;29(1):30-5.

47. Rajendran A, Parthsarathy S, Tamilselvan B, Seshadri KG, Shuaib M. Prevalence and correlates of disordered sleep in southeast asian indians with type 2 diabetes. Diabetes Metab J. 2012;36(1):70-6.

48. Cho EH, Lee H, Ryu OH, Choi MG, Kim SW. Sleep disturbances and glucoregulation in patients with type 2 diabetes. J Korean Med Sci. 2014;29(2):243-7.

49. Hung HC, Yang YC, Ou HY, Wu JS, Lu FH, Chang CJ. The relationship between impaired fasting glucose and self-reported sleep quality in a Chinese population. Clin Endocrinol. 2013;78(4):518-24.

50. Trento M, Broglio F, Riganti F, Basile M, Borgo E, Kucich $\mathrm{C}$, et al. Sleep abnormalities in type 2 diabetes may be associated with glycemic control. Acta Diabetol. 2008;45(4):225-9.

51. Tsujimura T, Matsuo Y, Keyaki T, Sakurada K, Imanishi J. Correlations of sleep disturbance with the immune system in type 2 diabetes mellitus. Diabetes Res Clin Pract. 2009;85(3):286-92.

52. Stamatakis KA, Punjabi NM. Effects of sleep fragmentation on glucose metabolism in normal subjects. Chest. 2010;137(1):95-101.

53. Meisinger C, Heier M, Loewel H, Study MKAC. Sleep disturbance as a predictor of type 2 diabetes mellitus in men and women from the general population. Diabetologia. 2005;48(2):235-41.

54. Suarez EC. Self-reported symptoms of sleep disturbance and inflammation, coagulation, insulin resistance and psychosocial distress: evidence for gender disparity. Brain Behav Immun. 2008;22(6):960-8.

55. St-Onge MP, Zammit G, Reboussin DM, Kuna ST, Sanders MH, Millman R, et al. Associations of sleep disturbance and duration with metabolic risk factors in obese persons with type 2 diabetes: data from the Sleep AHEAD Study. Nat Sci Sleep. 2012;4:143-50.

56. Harada Y, Oga T, Chin K, Takegami M, Takahashi K, Sumi $\mathrm{K}$, et al. Differences in relationships among sleep apnoea, glucose level, sleep duration and sleepiness between persons with and without type 2 diabetes. J Sleep Res. 2012;21(4):410-8.

57. Chasens ER, Korytkowski M, Sereika SM, Burke LE. Effect of poor sleep quality and excessive daytime sleepiness on factors associated with diabetes self-management. Diabetes Educ. 2013;39(1):74-82. 
58. Pallayova M, Donic V, Gresova S, Peregrim I, Tomori Z. Do differences in sleep architecture exist between persons with type 2 diabetes and nondiabetic controls? J Diabetes Sci Technol. 2010;4(2):344-52.

59. Nilsson PM, Roost M, Engstrom G, Hedblad B, Berglund G. Incidence of diabetes in middle-aged men is related to sleep disturbances. Diabetes Care. 2004;27(10):2464-9.

60. De Bacquer D, Van Risseghem M, Clays E, Kittel F, De Backer G, Braeckman L. Rotating shift work and the metabolic syndrome: a prospective study. Int J Epidemiol. 2009;38(3):848-54.

61. Buxton OM, Cain SW, O'Connor SP, Porter JH, Duffy JF, Wang W, et al. Adverse metabolic consequences in humans of prolonged sleep restriction combined with circadian disruption. Sci Transl Med. 2012;4(129):129ra43.

62. Reutrakul S, Hood MM, Crowley SJ, Morgan MK, Teodori M, Knutson KL. The relationship between breakfast skipping, chronotype, and glycemic control in type 2 diabetes. Chronobiol Int. 2014;31(1):64-71.

63. Reutrakul S, Hood MM, Crowley SJ, Morgan MK, Teodori M, Knutson KL, et al. Chronotype is independently associated with glycemic control in type 2 diabetes. Diabetes Care. 2013;36(9):2523-9.

64. Osonoi Y, Mita T, Osonoi T, Saito M, Tamasawa A, Nakayama $S$, et al. Morningness-eveningness questionnaire score and metabolic parameters in patients with type 2 diabetes mellitus. Chronobiol Int. 2014;31(9):1017-23.

65. Iwasaki M, Hirose $\mathrm{T}$, Mita $\mathrm{T}$, Sato $\mathrm{F}$, Ito $\mathrm{C}$, Yamamoto $\mathrm{R}$, et al. Morningness-eveningness questionnaire score correlates with glycated hemoglobin in middle-aged male workers with type 2 diabetes mellitus. J Diabetes Investig. 2013;4(4):376-81.

66. Fang W, Li Z, Wu L, Cao Z, Liang Y, Yang H, et al. Longer habitual afternoon napping is associated with a higher risk for impaired fasting plasma glucose and diabetes mellitus in older adults: results from the Dongfeng-Tongji cohort of retired workers. Sleep Med. 2013;14(10):950-4.

67. Lam KB, Jiang CQ, Thomas GN, Arora T, Zhang WS, Taheri S, et al. Napping is associated with increased risk of type 2 diabetes: the Guangzhou Biobank Cohort Study. Sleep. 2010;33(3):402-7.

68. Xu Q, Song Y, Hollenbeck A, Blair A, Schatzkin A, Chen H. Day napping and short night sleeping are associated with higher risk of diabetes in older adults. Diabetes Care. 2010;33(1):78-83.
69. Byberg S, Hansen AL, Christensen DL, Vistisen D, Aadahl M, Linneberg A, et al. Sleep duration and sleep quality are associated differently with alterations of glucose homeostasis. Diabet Med J Br Diabet Assoc. 2012;29(9):e354-60.

70. Arora T, Jiang CQ, Thomas GN, Lam KB, Zhang WS, Cheng KK, et al. Self-reported long total sleep duration is associated with metabolic syndrome: the Guangzhou Biobank Cohort Study. Diabetes Care. 2011;34(10):2317-9.

71. Rehman JU, Brismar K, Holmback U, Akerstedt T, Axelsson J. Sleeping during the day: effects on the 24-h patterns of IGF-binding protein 1, insulin, glucose, cortisol, and growth hormone. Eur J Endocrinol Eur Fed Endocr Soc. 2010;163(3):383-90.

72. Raman R, Gupta A, Venkatesh K, Kulothungan V, Sharma T. Abnormal sleep patterns in subjects with type II diabetes mellitus and its effect on diabetic microangiopathies: Sankara Nethralaya Diabetic Retinopathy Epidemiology and Molecular Genetic Study (SN-DREAMS, report 20). Acta Diabetol. 2012;49(4):255-61.

73. Killick R, Hoyos CM, Melehan KL, Dungan GC 2nd, Poh J, Liu PY. Metabolic and hormonal effects of 'catch-up' sleep in men with chronic, repetitive, lifestyle-driven sleep restriction. Clin Endocrinol. 2015;83(4):498-507.

74. Al-Delaimy WK, Manson JE, Willett WC, Stampfer $\mathrm{MJ}$, $\mathrm{Hu} \mathrm{FB}$. Snoring as a risk factor for type II diabetes mellitus: a prospective study. Am J Epidemiol. 2002;155(5):387-93.

75. Guest JF, Panca M, Sladkevicius E, Taheri S, Stradling J. Clinical outcomes and cost-effectiveness of continuous positive airway pressure to manage obstructive sleep apnea in patients with type 2 diabetes in the U.K. Diabetes Care. 2014;37(5):1263-71.

76. Pallayova M, Banerjee D, Taheri S. Novel insights into metabolic sequelae of obstructive sleep apnoea: a link between hypoxic stress and chronic diabetes complications. Diabetes Res Clin Pract. 2014;104(2):197-205.

77. Banerjee D, Leong WB, Arora T, Nolen M, Punamiya $\mathrm{V}$, Grunstein R, et al. The potential association between obstructive sleep apnea and diabetic retinopathy in severe obesity-the role of hypoxemia. PLoS One. 2013;8(11):e79521.

78. Leong WB, Banerjee D, Nolen M, Adab P, Thomas GN, Taheri S. Hypoxemia and glycemic control in type 2 diabetes mellitus with extreme obesity. J Clin Endocrinol Metab. 2014;99(9):E1650-4. 
79. Leong WB, Jadhakhan F, Taheri S, Chen YF, Adab P, Thomas GN. Effect of obstructive sleep apnoea on diabetic retinopathy and maculopathy: a systematic review and meta-analysis. Diabet Med J Br Diabet Assoc. 2015. doi:10.1111/dme.12817

80. Leong WB, Jadhakhan F, Taheri S, Thomas GN, Adab P. The association between obstructive sleep apnea on diabetic kidney disease: a systematic review and meta-analysis. Sleep. 2015; sp-00037-15.

81. Leong WB, Nolen M, Thomas GN, Adab P, Banerjee $D$, Taheri S. The impact of hypoxemia on nephropathy in extremely obese patients with type 2 diabetes mellitus. J Clin Sleep Med JCSM Off Publ Am Acad Sleep Med. 2014;10(7):773-8.

82. Leong WB, Arora T, Jenkinson D, Thomas A, Punamiya V, Banerjee $\mathrm{D}$, et al. The prevalence and severity of obstructive sleep apnea in severe obesity: the impact of ethnicity. J Clin Sleep Med JCSM Off Publ Am Acad Sleep Med. 2013;9(9):853-8.

83. Resnick HE, Redline S, Shahar E, Gilpin A, Newman A, Walter R, et al. Diabetes and sleep disturbances: findings from the Sleep Heart Health Study. Diabetes Care. 2003;26(3):702-9.

84. Tasali E, Leproult R, Ehrmann DA, Van Cauter E. Slow-wave sleep and the risk of type 2 diabetes in humans. Proc Natl Acad Sci USA. 2008;105(3):1044-9.

85. Spiegel K, Tasali E, Penev P, Van Cauter E. Brief communication: sleep curtailment in healthy young men is associated with decreased leptin levels, elevated ghrelin levels, and increased hunger and appetite. Ann Intern Med. 2004;141(11):846-50.

86. Taheri S, Lin L, Austin D, Young T, Mignot E. Short sleep duration is associated with reduced leptin, elevated ghrelin, and increased body mass index. PLoS Med. 2004;1(3):e62.

87. Nedeltcheva AV, Kilkus JM, Imperial J, Kasza K, Schoeller DA, Penev PD. Sleep curtailment is accompanied by increased intake of calories from snacks. Am J Clin Nutr. 2009;89(1):126-33.

88. Taheri S, Bloom S. Orexins/hypocretins: waking up the scientific world. Clin Endocrinol. 2001;54(4):421-9.

89. Taheri S, Hafizi S. The orexins/hypocretins: hypothalamic peptides linked to sleep and appetite. Psychol Med. 2002;32(6):955-8.

90. Taheri S, Ward H, Ghatei M, Bloom S. Role of orexins in sleep and arousal mechanisms. Lancet. 2000;355(9206):847.
91. Chamarthi B, Gaziano JM, Blonde L, Vinik A, Scranton RE, Ezrokhi $M$, et al. Timed Bromocriptine-QR therapy reduces progression of cardiovascular disease and dysglycemia in subjects with well-controlled type 2 diabetes mellitus. J Diabetes Res. 2015;2015:157698.

92. Roe ED, Chamarthi B, Raskin P. Impact of Bromocriptine-QR therapy on glycemic control and daily insulin requirement in type 2 diabetes mellitus subjects whose dysglycemia is poorly controlled on high-dose insulin: a Pilot Study. J Diabetes Res. 2015;2015:834903.

93. Gonzalez S, Moreno-Delgado D, Moreno E, Perez-Capote K, Franco R, Mallol J, et al. Circadian-related heteromerization of adrenergic and dopamine $\mathrm{D}(4)$ receptors modulates melatonin synthesis and release in the pineal gland. PLoS Biol. 2012;10(6):e1001347.

94. Shan Z, Ma H, Xie M, Yan P, Guo Y, Bao W, et al. Sleep duration and risk of type 2 diabetes: a meta-analysis of prospective studies. Diabetes Care. 2015;38(3):529-37.

95. Lesser H, Sharma U, LaMoreaux L, Poole RM. Pregabalin relieves symptoms of painful diabetic neuropathy: a randomized controlled trial. Neurology. 2004;63(11):2104-10.

96. Leproult R, Deliens G, Gilson M, Peigneux P. Beneficial impact of sleep extension on fasting insulin sensitivity in adults with habitual sleep restriction. Sleep. 2015;38(5):707-15.

97. Barone MT, Wey D, Schorr F, Franco DR, Carra MK, Lorenzi Filho G, et al. Sleep and glycemic control in type 1 diabetes. Arch Endocrinol Metab. 2015;59(1):71-8.

98. Feupe SF, Frias PF, Mednick SC, McDevitt EA, Heintzman ND. Nocturnal continuous glucose and sleep stage data in adults with type 1 diabetes in real-world conditions. J Diabetes Sci Technol. 2013;7(5):1337-45.

99. Rafalson L, Donahue RP, Stranges S, Lamonte MJ, Dmochowski J, Dorn J, et al. Short sleep duration is associated with the development of impaired fasting glucose: the Western New York Health Study. Ann Epidemiol. 2010;20(12):883-9.

100. Nakanishi-Minami T, Kishida K, Funahashi T, Shimomura I. Sleep-wake cycle irregularities in type 2 diabetics. Diabetol Metab Syndr. 2012;4(1):18.

101. Liu A, Kushida CA, Reaven GM. Habitual shortened sleep and insulin resistance: an independent 
relationship in obese individuals. Metab Clin Exp. 2013;62(11):1553-6.

102. Inkster B, Riha RL, Van Look L, Williamson R, McLachlan S, Frier BM, et al. Association between excessive daytime sleepiness and severe hypoglycemia in people with type 2 diabetes: the Edinburgh Type 2 Diabetes Study. Diabetes Care. 2013;36(12):4157-9.

103. Ohkuma T, Fujii H, Iwase M, Ogata-Kaizu S, Ide H, Kikuchi Y, et al. U-shaped association of sleep duration with metabolic syndrome and insulin resistance in patients with type 2 diabetes: the Fukuoka Diabetes Registry. Metab Clin Exp. 2014;63(4):484-91.

104. Pyykkonen AJ, Isomaa B, Pesonen AK, Eriksson JG, Groop L, Tuomi T, et al. Sleep duration and insulin resistance in individuals without type 2 diabetes: the PPP-Botnia study. Ann Med. 2014;46(5):324-9.

105. Zheng Y, Wang A, Pan C, Lu J, Dou J, Lu Z, et al. Impact of night sleep duration on glycemic and triglyceride levels in Chinese with different glycemic status. J Diabetes. 2015;7(1):24-30.

106. Baoying H, Hongjie C, Changsheng Q, Peijian W, Qingfei L, Yinghua L, et al. Association of napping and night-time sleep with impaired glucose regulation, insulin resistance and glycated haemoglobin in Chinese middle-aged adults with no diabetes: a cross-sectional study. BMJ Open. 2014;4(7):e004419.

107. Olsson L, Ahlbom A, Grill V, Midthjell K, Carlsson S. Sleep disturbances and low psychological well-being are associated with an increased risk of autoimmune diabetes in adults. Results from the Nord-Trondelag Health Study. Diabetes Res Clin Pract. 2012;98(2):302-11.

108. Schmid SM, Hallschmid M, Jauch-Chara K, Bandorf N, Born J, Schultes B. Sleep loss alters basal metabolic hormone secretion and modulates the dynamic counterregulatory response to hypoglycemia. J Clin Endocrinol Metab. 2007;92(8):3044-51.

109. Tuomilehto H, Peltonen M, Partinen M, Lavigne G, Eriksson JG, Herder C, et al. Sleep duration, lifestyle intervention, and incidence of type 2 diabetes in impaired glucose tolerance: the Finnish Diabetes Prevention Study. Diabetes Care. 2009;32(11):1965-71.

110. Nedeltcheva AV, Kessler L, Imperial J, Penev PD. Exposure to recurrent sleep restriction in the setting of high caloric intake and physical inactivity results in increased insulin resistance and reduced glucose tolerance. J Clin Endocrinol Metab. 2009;94(9):3242-50.

111. van Leeuwen WM, Hublin C, Sallinen M, Harma M, Hirvonen A, Porkka-Heiskanen T. Prolonged sleep restriction affects glucose metabolism in healthy young men. Int J Endocrinol. 2010;2010:108641.

112. Garfinkel D, Zorin M, Wainstein J, Matas Z, Laudon M, Zisapel N. Efficacy and safety of prolonged-release melatonin in insomnia patients with diabetes: a randomized, double-blind, crossover study. Diabetes Metab Syndr Obes Targets Ther. 2011;4:307-13.

113. Bell LN, Kilkus JM, Booth JN 3rd, Bromley LE, Imperial JG, Penev PD. Effects of sleep restriction on the human plasma metabolome. Physiol Behav. 2013;122:25-31.

114. Robertson MD, Russell-Jones D, Umpleby AM, Dijk DJ. Effects of three weeks of mild sleep restriction implemented in the home environment on multiple metabolic and endocrine markers in healthy young men. Metab Clin Exp. 2013;62(2):204-11.

115. Gonzalez-Ortiz M, Martinez-Abundis E, Balcazar-Munoz BR, Pascoe-Gonzalez S. Effect of sleep deprivation on insulin sensitivity and cortisol concentration in healthy subjects. Diabetes Nutr Metab. 2000;13(2):80-3.

116. VanHelder T, Symons JD, Radomski MW. Effects of sleep deprivation and exercise on glucose tolerance. Aviat Space Environ Med. 1993;64(6):487-92.

117. Buxton OM, Pavlova M, Reid EW, Wang W, Simonson DC, Adler GK. Sleep restriction for 1 week reduces insulin sensitivity in healthy men. Diabetes. 2010;59(9):2126-33. 